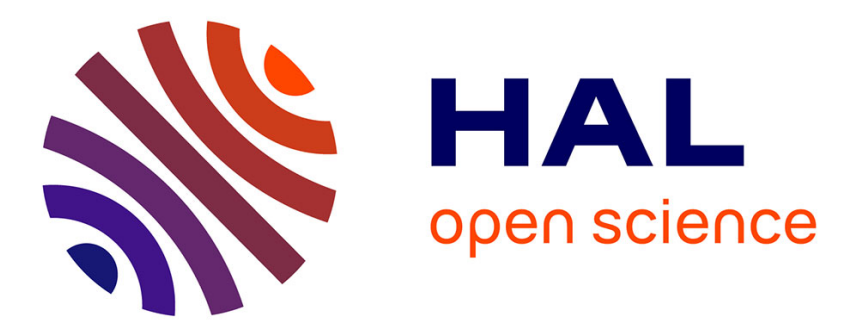

\title{
Exploring Green Solvents Associated to Pd/C as Heterogeneous Catalyst for Direct Arylation of Heteroaromatics with Aryl Bromides
}

\author{
Shuxin Mao, Xinzhe Shi, Jean-François Soulé, Henri Doucet
}

\section{- To cite this version:}

Shuxin Mao, Xinzhe Shi, Jean-François Soulé, Henri Doucet. Exploring Green Solvents Associated to $\mathrm{Pd} / \mathrm{C}$ as Heterogeneous Catalyst for Direct Arylation of Heteroaromatics with Aryl Bromides. Advanced Synthesis and Catalysis, 2018, 360 (17), pp.3306-3317. 10.1002/adsc.201800596 . hal01874561

HAL Id: hal-01874561

https://hal-univ-rennes1.archives-ouvertes.fr/hal-01874561

Submitted on 20 Sep 2018

HAL is a multi-disciplinary open access archive for the deposit and dissemination of scientific research documents, whether they are published or not. The documents may come from teaching and research institutions in France or abroad, or from public or private research centers.
L'archive ouverte pluridisciplinaire HAL, est destinée au dépôt et à la diffusion de documents scientifiques de niveau recherche, publiés ou non, émanant des établissements d'enseignement et de recherche français ou étrangers, des laboratoires publics ou privés. 


\title{
Exploring Green Solvents Associated to $\mathrm{Pd} / \mathrm{C}$ as Heterogeneous Catalyst for Direct Arylation of Heteroaromatics with Aryl Bromides
}

\author{
Shuxin Mao, ${ }^{[a]}$ Xinzhe Shi, ${ }^{[a]}$ Jean-François Soulé ${ }^{\star[a]}$ and Henri Doucet ${ }^{\star[a]}$
}

\begin{abstract}
Metal residues are certainly one of the major sources of contamination of products in metal-catalyzed direct arylation reactions. We found that the use of only $1 \mathrm{~mol} \%$ of the heterogeneous catalyst $\mathrm{Pd} / \mathrm{C}$ promotes very efficiently the direct arylations of most heteroaromatics. In the presence of this catalyst and potassium acetate as the base, the direct arylation of thiophenes, furans, pyrroles, thiazoles, imidazoles or isoxazoles, using aryl bromides as coupling partners, proceeds highly regioselectively and in moderate to very high yields. With several heteroarenes both electron-deficient and electron-rich aryl bromides were tolerated; moreover, with the most reactive heteroarenes, the $\mathrm{Pd} / \mathrm{C}$ catalyst tolerated green solvents such as diethyl carbonate, 3methylbutan-1-ol and pentan-1-ol, affording a synthetic scheme with low environmental impact.
\end{abstract}

\section{Introduction}

The access to arylated heteroaromatics is a very important research field in organic synthesis due to the physical or biological properties of such derivatives. For example, Dantrolene, Canagliflozin, Zolpidem, Atorvastatin, and Valdecoxib are currently employed for the treatment of various diseases (Figure 1).

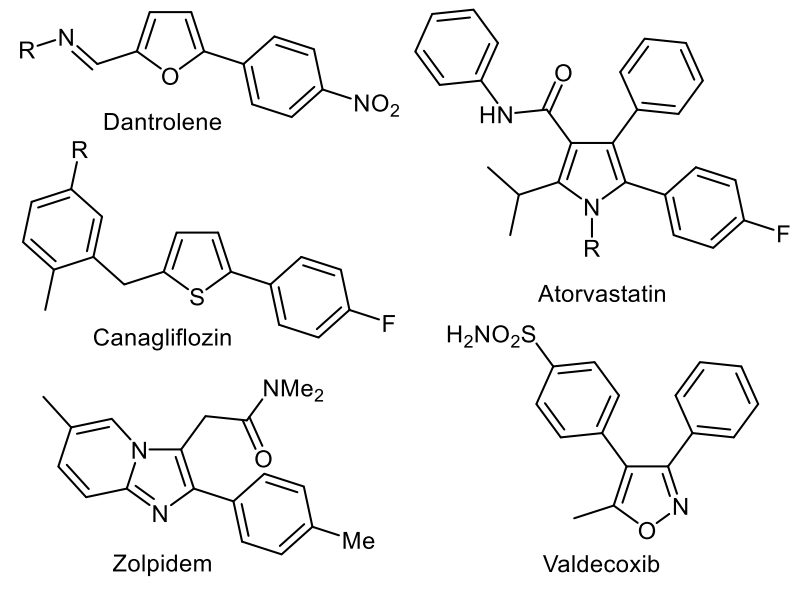

Figure 1. Examples of pharmaceuticals containing an arylated heteroaromatic

In 1990, Ohta et al. discovered that the arylation of several heteroaromatics using aryl halides as aryl source proceed via

[a] Mr Shuxin Mao, Mrs Xinzhe Shi, Dr. Jean-François Soulé, Dr. Henri Doucet

Univ Rennes, CNRS, ISCR-UMR 6226, F-35000 Rennes, France. E-mail: jean-francois.soule@univ-rennes1.fr; henri.doucet@univrennes1.fr

Supporting information for this article is given via a link at the end of the document. the $\mathrm{C}-\mathrm{H}$ bond activation of heteroaromatics using $\mathrm{Pd}\left(\mathrm{PPh}_{3}\right)_{4}$ catalyst. ${ }^{[1]}$ Since these seminal results, the so-called $\mathrm{Pd}$ catalyzed "direct arylation" of heteroarenes was demonstrated to be an extremely powerful method for the preparation of a wide variety of arylated heterocycles. ${ }^{[2,3]}$ This methodology is environmentally attractive, as the major reaction by-product is $\mathrm{HX}$ associated to a base, instead of the metallic salts mixture which is produced under the more classical Suzuki, Negishi or Stille cross-coupling reactions. ${ }^{[4]}$ However, in most cases, direct arylations are currently performed using homogeneous palladium-catalysts, producing metal and ligands residues, which are a major issue in the pharmaceutical chemistry and also for some materials possessing specific optical properties. Moreover, these homogeneous catalysts are generally associated with relatively toxic solvents such as DMA, DMF, NMP, or dioxane..$^{[5-10]}$

In recent years, a few solvents which can be considered as "green"[11] according to P. Anastas principles, have been employed for Pd-catalysed direct arylations of heteroaromatics. ${ }^{[12-17]}$ For example, water was employed by Greaney and Djakovitch for the arylation of some 5-membered ring heterocycles; ${ }^{[13]}$ whereas, Ackermann employed a polyethylene glycol solvent for the direct arylation of triazoles. ${ }^{[14]}$ Carbonates, ethers or alcohols have been used as the solvents for the arylation of heteroarenes. ${ }^{[15-17]}$ All these reactions were performed using homogeneous Pd-catalysts.

A few examples of $\mathrm{Pd}$-catalyzed direct arylations of heteroarenes using heterogeneous palladium catalysts have been described. ${ }^{[18-24]}$ The first one was reported in 1982 and employed $\mathrm{Pd} / \mathrm{C}$ catalyst, but it was limited to isoxazoles as heteroarene and HMPT, which is considered as a toxic solvent, was used.[20] In 2013, Glorius et al. reported the heterogeneously catalyzed direct $\beta$-arylation of benzothiophenes with aryl chlorides using $\mathrm{Pd} / \mathrm{C}$ catalyst in the presence of 10 $\mathrm{mol} \% \mathrm{CuCl}_{2}{ }^{[21]}$ One year later, this group extended the use of $\mathrm{Pd} / \mathrm{C}$ catalyst to the C4-arylation of thiophenes using aryl iodonium salts as aryl source and ethanol or water/ethanol mixtures as green solvents. ${ }^{[22]}$ Only 1,2,3-triazole has been successfully employed in $\mathrm{Pd}$-catalyzed direct arylation with aryl halides as aryl source using a heterogeneous catalyst in a green solvent. ${ }^{[18]}$ For this reaction, Ackermann, Vaccaro et al. employed $\gamma$-valerolactone as renewable solvent associated with $5 \mathrm{~mol} \% \mathrm{Pd} / \mathrm{C}$. To our knowledge, the reactivity of other heteroaromatics and the influence of other "green" solvents using $\mathrm{Pd} / \mathrm{C}$ catalyst for direct arylations with aryl halides as aryl source has not been described.

The use of the heterogeneous catalyst $\mathrm{Pd} / \mathrm{C}$ presents several advantages: 1) it is not air or moisture sensitive and therefore easy to handle, 2) it can be easily removed from the product by simple filtration at the end of the reaction, 3) the product is contaminated with very low amount of Pd residues, 4) there is no phosphine residues, 5 ) it is easily available at an affordable 
cost, and 6) recycling of the recovered $\mathrm{Pd} / \mathrm{C}$ is generally possible.

Herein, we report on the use of $\mathrm{Pd} / \mathrm{C}$ for the direct arylations of a wide range of heteroaromatic derivatives with aryl bromides using, in several cases, solvents which can be considered as "green" and which are available on large scale at an affordable cost (Figure 2):

1) diethyl carbonate (DEC) which displays several advantages such as a high biodegradability, low acute toxicity and a low environmental impact in the course of its synthesis as it can be prepared from $\mathrm{CO}_{2}$ and bioethanol;[25]

2) cyclopentyl methyl ether (CPME) which also presents several advantageous features such as limited miscibility in water which allows easy separation, low formation of peroxides (compared with THF or diisopropyl ether). Moreover, CPME can be manufactured by the addition of $\mathrm{MeOH}$ to cyclopentene which produces no apparent waste; ;26]

3) 3-methylbutan-1-ol and pentan-1-ol which are biodegradable, are not considered as hazardous air pollutant solvents, are unlikely to have any adverse health effects, as they are found in several alcoholic beverages, and can be prepared by fermentation or by the reduction of 1-valeraldehyde or 3methylbutyraldehyde with hydrogen.

$$
\begin{gathered}
\begin{array}{c}
\text { CycloPentyl } \\
\text { Methyl Ether } \\
(\mathrm{CPME})
\end{array} \\
\begin{array}{c}
\text { DiEthyl Carbonate } \\
(\mathrm{DEC})
\end{array} \\
\text { 3-Methylbutan-1-ol Pentan-1-ol }
\end{gathered}
$$

Figure 2. Green solvents employed for $\mathrm{Pd} / \mathrm{C}$ catalyzed arylations

\section{Results and Discussion}

The first set of reactions using 2- $n$-pentylthiophene and 4bromobenzonitrile as coupling partners was carried out under previously reported reaction conditions, but using $1 \mathrm{~mol} \%$ of $10 \% \mathrm{Pd} / \mathrm{C}$ as the catalyst (Scheme 1). ${ }^{[5 \mathrm{a}]}$ In the presence of the polar solvent $\mathrm{N}, \mathrm{N}$-dimethylacetamide (DMA), a high yield in the desired C5-arylated thiazole 1 was obtained. No formation of other arylated or diarylated thiophenes was detected by GC/MS analysis. As DMA is not a desirable solvent in terms of "green chemistry", the outcome of this reaction using four "greener" solvents was studied. The reaction in DEC or CPME with 1 mol\% of $10 \% \mathrm{Pd} / \mathrm{C}$ catalyst afforded 1 in only $21 \%$ and $<5 \%$ yields, respectively, due to partial conversions of 4bromobenzonitrile. Conversely, 3-methylbutan-1-ol and pentan1-ol afforded 1 in high yields with complete conversions of the aryl bromide.

Then, a set of aryl bromides was reacted with 2- $n$ pentylthiophene using $1 \mathrm{~mol} \%$ of $10 \% \mathrm{Pd} / \mathrm{C}$ catalyst. High yields were generally obtained for the coupling of 2- $n$-pentylthiophene with aryl bromides in DMA. Both electron-withdrawing and electron-donating substituents were tolerated. It should be mentioned that for the reaction with 4-bromonitrobenzene, a higher yield in $\mathbf{4}$ was obtained using 3-methylbutan-1-ol instead of DMA as the solvent, due to the formation of a lower amount of

side-products. A high yield in 7 was also obtained for the reaction of 2-bromobenzonitrile in 3-methylbutan-1-ol. Thiophene 2-carbonitrile also reacted nicely with 4bromobenzonitrile and 4-bromoacetophenone using $1 \mathrm{~mol} \%$ of $10 \% \mathrm{Pd} / \mathrm{C}$ catalyst in DMA affording 8 and 9 in $87 \%$ and $90 \%$ yields, respectively. Then, thiophenes substituted by ester or acetyl functions at $\mathrm{C} 2$ position were employed. In both cases, high yields in the expected coupling products 10 and 11 were obtained. 2-n-Pentylthiophene also underwent C5-arylation with 3 -bromopyridine to give product 12 in $90 \%$ yield. The use of $p$ tolylboronic acid as aryl source instead of 4-bromobenzene for the preparation of $\mathbf{5}$, under the same conditions, but using $\mathrm{Cu}(\mathrm{OAc})_{2}$ as oxidant gave no coupling product.
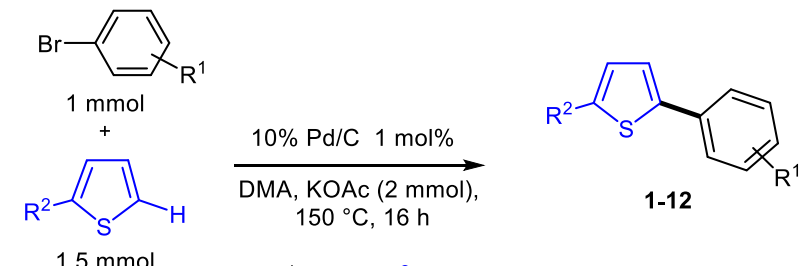

$1.5 \mathrm{mmol}$

$$
\begin{aligned}
& \begin{array}{llll}
\mathrm{R}^{1} & \mathrm{R}^{2} & & \text { Yield (\%) } \\
\text { 4-CN } & n \text { Pent } & \mathbf{1} & 91,84^{*}, 81^{*}
\end{array} \\
& \text { 4-COMe nPent } 291 \\
& \text { 4- } \mathrm{CO}_{2} \mathrm{Et} \text { nPent } \mathbf{3} \text { 88, 54* } \\
& 4-\mathrm{NO}_{2} \quad n \text { Pent } 4 \text { 26, 67* } \\
& \text { 4-Me } \quad n \text { Pent } \mathbf{5} 90,0^{* * * * *} \\
& \text { 4-MeO } n \text { Pent } 6 \quad 76 \\
& \text { 2-CN } n \text { Pent } 7 \text { 93* } \\
& 4-\mathrm{CN} C \mathrm{CN} 887,41^{*}, 21^{\text {***}} \\
& \text { 4-COMe CN } 990 \\
& \text { 4-CN COMe } 1079 \\
& \text { 4-CN } \quad \mathrm{CO}_{2} \text { Et } 11 \quad 84
\end{aligned}
$$

\footnotetext{
*: Reaction in 3-methylbutan-1-ol

**: Reaction in pentan-1-ol

$* * *$ : Reaction in diethylcarbonate

$* * * *$ : Reaction in cyclopentyl methyl ether

$* * * *$ Reaction in cyclopentyl methyl ether

with $2 \mathrm{mmol}$ of $\mathrm{Cu}(\mathrm{OAc})_{2}$
}

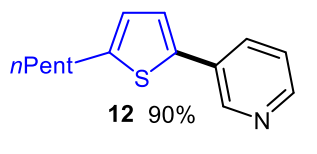

Scheme 1. Pd/C catalyzed coupling of thiophene derivatives with aryl bromides.

The reactivity of several furan derivatives in the presence of 1 $\mathrm{mol} \% \mathrm{Pd} / \mathrm{C}$ catalyst was also examined (Scheme 2). The arylation of furan derivatives had been previously reported to proceed nicely in DMA using $\operatorname{PdCl}\left(\mathrm{C}_{3} \mathrm{H}_{5}\right)(\mathrm{dppb})$ as the catalyst. ${ }^{[6 d]} \quad$ A poor reactivity of 2-n-butylfuran with 4bromobenzonitrile was observed in DEC; whereas the reactions performed in 3-methylbutan-1-ol or DMA gave the desired product 13 in $43 \%$ and $71 \%$ yields, respectively. The arylation of 2 - $n$-butylfuran in DMA using $1 \mathrm{~mol} \%$ of $10 \% \mathrm{Pd} / \mathrm{C}$ catalyst also tolerated 4-acetyl- 4-methyl- or 2-cyano-substituents on the aryl bromide affording $\mathbf{1 4 - 1 6}$ in $67-88 \%$ yields. A good yield in product 17 was also obtained for the reaction with 3bromopyridine. Under the same conditions, the reaction of 2 ethylbenzofuran with 4-bromobenzonitrile gave 18 in $83 \%$ yield. 


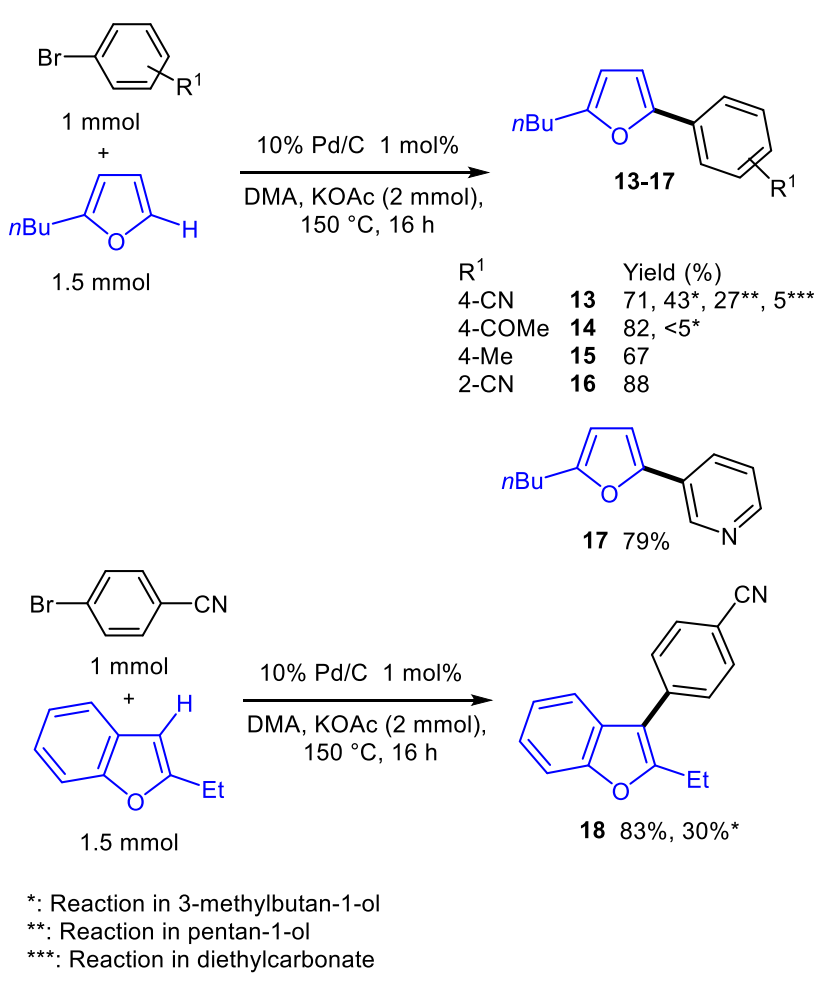

Scheme 2. $\mathrm{Pd} / \mathrm{C}$ catalyzed coupling of (benzo)furan derivatives with aryl bromides.

The use of $1 \mathrm{~mol} \%$ of $10 \% \mathrm{Pd} / \mathrm{C}$ catalyst for the C2-arylation of 1-methylpyrrole was also successful; however, for these reactions, a larger excess of the heteroarene (4 equiv.) was employed in order to avoid the formation of 2,5-diarylated pyrroles as side-products (Scheme 3). With 2- and 4bromobenzonitriles, good yields in the desired coupling products 19 and 22 were obtained in DMA and in 3-methylbutan-1-ol. Conversely, a moderate yield of $\mathbf{2 0}$ was obtained for the reaction of 1-methylpyrrole with ethyl 4-bromobenzoate in 3-methylbutan1-ol, due to the formation of degradation products. With this aryl bromide, the use of DMA as the solvent allowed to obtain 20 in a higher yield. The reaction of 1-methylpyrrole with 4bromotoluene gave the target product $\mathbf{2 1}$ in only $52 \%$ yield, as large amount of $4,4^{\prime}$-dimethyl-1,1'-biphenyl arising from the homo-coupling of 4-bromotoluene was also produced.

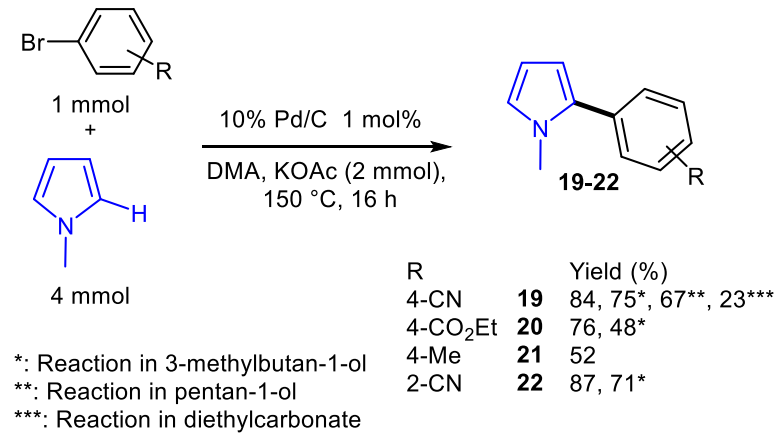

Scheme 3. Pd/C catalyzed coupling of pyrrole derivatives with aryl bromides.

Then, we examined the reactivity of thiazoles with a set of aryl bromides in the presence of $1 \mathrm{~mol} \%$ of $10 \% \mathrm{Pd} / \mathrm{C}$. Firstly, various solvents were investigated for the arylation of 2isopropyl-4-methylthiazole with 4-bromobenzonitrile using $\mathrm{Pd} / \mathrm{C}$ as heterogeneous catalyst (Scheme 4). In the presence of the polar solvent DMA, a very high yield into the desired C5-arylated thiazole $\mathbf{2 3}$ was obtained. Then, the outcome of this reaction using four "greener" solvents was studied. The reaction using, CPME and $1 \mathrm{~mol} \%$ of $10 \% \mathrm{Pd} / \mathrm{C}$ catalyst afforded 23 in only $48 \%$ yield due to a partial conversion of 4 -bromobenzonitrile. Conversely, DEC, 3-methylbutan-1-ol and pentan-1-ol gave 23 in very high yields (94-97\%) with complete conversions of the aryl bromide. The yield in $\mathbf{2 3}$ was very similar for a reaction performed on a larger scale $(5 \mathrm{mmol}$ of 2-isopropy-4methylthiazole instead of $1 \mathrm{mmol}$ ) in $10 \mathrm{~mL}$ of 3-methylbutan-1ol.

The reaction of several other para-substituted electron-deficient aryl bromides such as 4-bromobenzaldehyde, 4bromoacetophenone, ethyl 4-bromobenzoate or 4bromonitrobenzene with 2-isopropy-4-methylthiazole in 3methylbutan-1-ol using $1 \mathrm{~mol} \%$ of $10 \% \mathrm{Pd} / \mathrm{C}$ also gave the desired products 24-27 in very high yields. 4Fluorobromobenzene afforded the coupling product 28 in a higher yield in DEC (97\%) than in 3-methylbutan-1-ol (85\%). The presence of the electron-donating para-substituents, methyl, $t$-butyl and methoxy on the aryl bromide was also tolerated providing 29-31 in 72-92\% yields. The reaction of 2-isopropy-4methylthiazole with 4-bromo- $\mathrm{N}, \mathrm{N}$-dimethylaniline in 3methylbutan-1-ol gave 32 in only $41 \%$ yields due to partial conversion of the aniline derivative; however, the use of DMA as the solvent allowed to increase the yield to $78 \%$. As expected, the reactivity of the meta-substituted 3,5bis(trifluoromethyl)bromobenzene was found to be similar to para-substituted aryl bromides. The reactivity of four orthosubstituted aryl bromides was also examined. Due to their coordination and/or steric properties, ortho-substituents on aryl halides may have an important influence on the yields of Pdcatalysed reactions. 2-Cyano-, 2-trifluoromethyl- and 2-fluorosubstituents were tolerated, affording $\mathbf{3 4}, \mathbf{3 6}$ and $\mathbf{3 7}$ in $85-94 \%$ yields, when the reactions were performed in 3-methylbutan-1-ol 
or in DEC. The reaction with 2-bromonitrobenzene gave 35 in lower yields due to the formation of several unidentified degradation products. This novel environmentally friendly protocol using DEC or 3-methylbutan-1-ol and $1 \mathrm{~mol} \%$ of $10 \%$ $\mathrm{Pd} / \mathrm{C}$ was also effective for the direct couplings of 2-isopropyl-4methylthiazole with 3-bromopyridine or 3-bromoquinoline. With these substrates, the target products $\mathbf{3 8}$ and $\mathbf{3 9}$ were obtained in $88-96 \%$ yields. 2-Ethyl-4-methylthiazole which is slightly less hindered than 2-isopropyl-4-methylthiazole exhibits a similar reactivity. Its reaction with 4-bromobenzonitrile and 4bromopropiophenone in 3-methylbutan-1-ol or DEC using 1 $\mathrm{mol} \% \mathrm{Pd} / \mathrm{C}$ gave $\mathbf{4 0}$ and $\mathbf{4 1}$ in $95-96 \%$ yields.
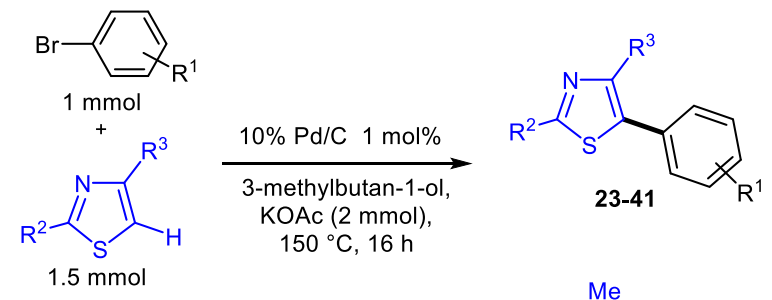<smiles>[R]c1ccc(-c2sc(C(C)C)nc2C)cc1</smiles><smiles>Cc1nc(C(C)C)sc1-c1cccnc1</smiles>

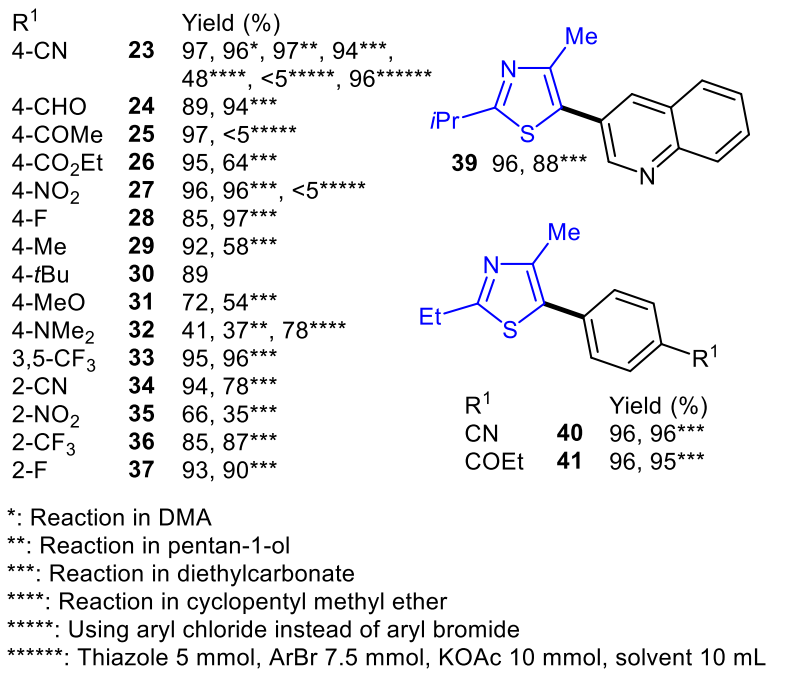

Scheme 4. $\mathrm{Pd} / \mathrm{C}$ catalyzed coupling of thiazole derivatives with aryl bromides.

We also studied the direct arylation of 1-methylimidazole using 1 $\mathrm{mol} \%$ of $10 \% \mathrm{Pd} / \mathrm{C}$ catalyst (Scheme 5 ). In the course of this reaction, both positions $\mathrm{C} 2$ and $\mathrm{C} 5$ of imidazole might have been arylated. Using our procedure, in the presence of 2- or 4bromobenzonitrile in DMA, a regioselective C5-arylation was observed, affording 42 and 44 in high yields. Moreover, good yields of target compounds 43 and 45 were obtained using the deactivated aryl bromide 4-bromotoluene, or a heteroarene.

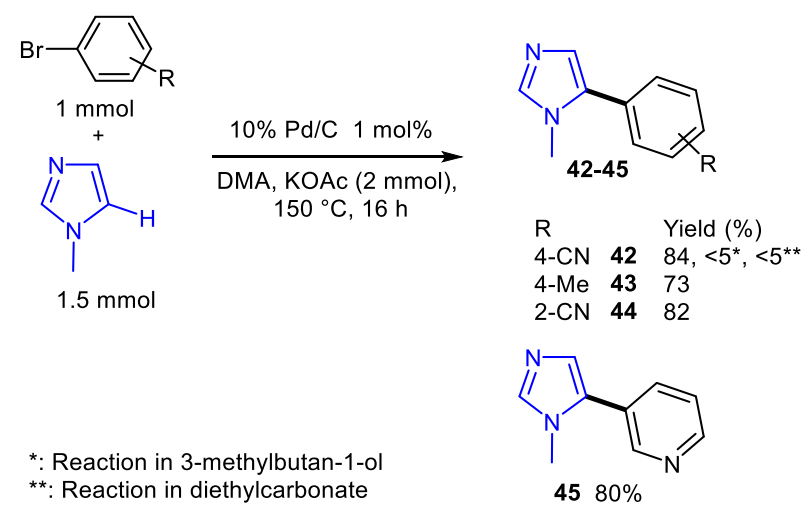

Scheme 5. Pd/C catalyzed coupling of 1-methylimidazole with aryl bromides.

4-Arylisoxazoles are important structures due to their specific biological properties. For example, Valdecoxib (Figure 1) and Parecoxib, which contain this unit, are nonsteroidal antiinflammatory drugs. The reaction of 3,5-dimethylisoxazole with 4-bromobenzonitrile in the presence of $1 \mathrm{~mol} \%$ of $10 \% \mathrm{Pd} / \mathrm{C}$ catalyst in DMA gave the C4-arylated isoxazole in $93 \%$ yield (Scheme 6). Similar yields were obtained for the coupling of 3,5-dimethylisoxazole with ethyl 4-bromobenzoate and 2bromobenzonitrile; whereas a lower yield in $\mathbf{4 8}$ was obtained with electron-rich 4-bromoanisole. The use of 3-methylbutan-1ol as solvent for the arylation of 3,5-dimethylisoxazole was ineffective.
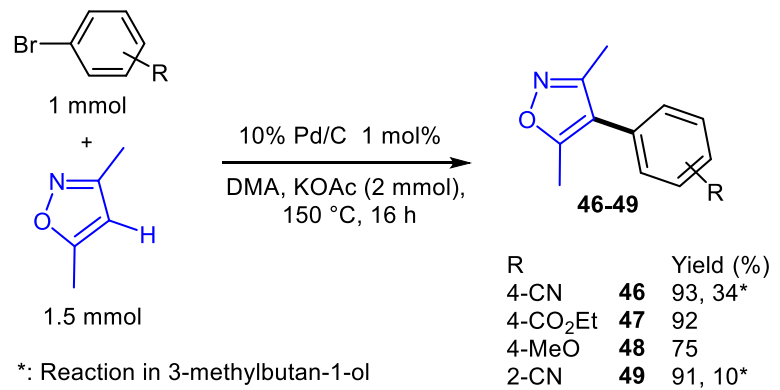

Scheme 6. Pd/C catalyzed coupling of 3,5-dimethylisoxazole with aryl bromides.

Among heterocycles, imidazo[1,2-a]pyridines and imidazo[1,2b]pyridazines display very important biological properties. For example, Zolpidem (Figure 1) is actually employed for the shortterm treatment of insomnia, Miroprofen is a nonsteroidal antiinflammatory drug and Ponatinib which contains an imidazo[1,2b]pyridazine unit is used for the treatment of leukemia. Therefore, the discovery of effective procedures for the direct coupling of these two heteroarenes with aryl bromides is important for biochemists. We first studied the reactivity of imidazo[1,2-a]pyridine with para-substituted aryl bromides, using $1 \mathrm{~mol} \%$ of $10 \% \mathrm{Pd} / \mathrm{C}$ catalyst in 3-methylbutan-1-ol. In all cases, very high yields of the expected coupling products 50-52 were 
obtained. A lower yield in $\mathbf{5 3}$ was obtained for the reaction with 2-bromobenzonitrile in 3-methylbutan-1-ol, but the use of DMA as solvent afforded 53 in $92 \%$ yield. 3-Bromoquinoline was found to be very reactive in 3-methylbutan-1-ol, as 54 was obtained in $94 \%$ yield. The reactivity of imidazo[1,2-b]pyridazine is quite similar, as in all cases including with 2-bromobenzonitrile and 4-bromoanisole, the desired products 55-60 were obtained in good yields using 3-methylbutan-1-ol as the solvent. Moreover, high yields in $\mathbf{5 5}$ and $\mathbf{5 6}$ were also obtained in DEC.
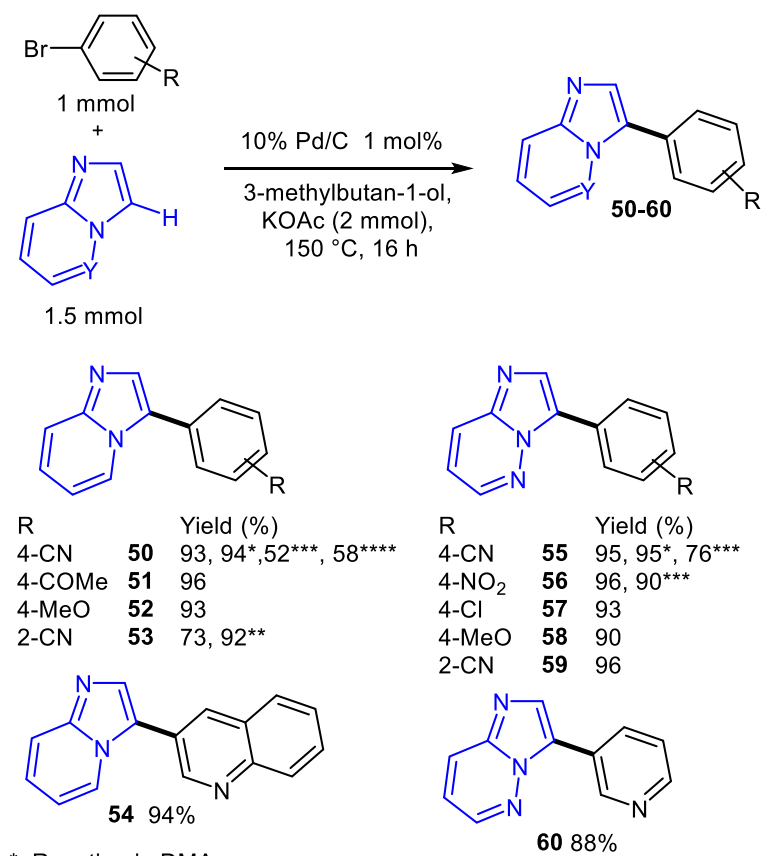

*: Reaction in DMA

**: Reaction in pentan-1-ol

$* * *$ : Reaction in diethylcarbonate

****: Reaction in cyclopentyl methyl ether

Scheme 7. Pd/C catalyzed coupling of imidazo[1,2-a]pyridine and imidazo[1,2b]pyridazine with aryl bromides.

The mechanism of such direct arylations using $\mathrm{Pd} / \mathrm{C}$ catalyst was not elucidated. ${ }^{[27]}$ In 2003, Conlon et al. reported that the Suzuki-Miyaura reaction using heterogeneous $\mathrm{Pd} / \mathrm{C}$ catalyst has a homogeneous component due to the formation of soluble $\mathrm{Pd}$ species during the reaction. ${ }^{[27 a]}$ They assumed that the desorption of palladium from $\mathrm{Pd} / \mathrm{C}$ occurs after oxidative addition of the aryl halide on the $\mathrm{Pd} / \mathrm{C}$ surface, which generates a soluble $\mathrm{Pd}$ (II) species. As the direct arylations reported here employ quite similar reaction conditions, a homogeneous component in the catalytic cycle is also possible.

\section{Conclusions}

In summary, we demonstrated that $\mathrm{Pd} / \mathrm{C}$ is an efficient catalyst for the direct arylation of a wide range of heteroaromatics. In the presence $1 \mathrm{~mol} \%$ of $10 \% \mathrm{Pd} / \mathrm{C}$, the direct arylation of thiazoles, thiophenes, furans, pyrroles, imidazoles or isoxazoles using aryl bromides as coupling partners proceeds highly regioselectively, and in moderate to very high yields. It should be mentioned that with several heteroarenes, both electron-deficient and electronrich aryl bromides were reactive under these conditions, and a wide range of functionalities such as acetyl, propionyl, formyl, ester, nitro, nitrile, trifluoromethyl, chloro, fluoro, methoxy or amino on the aryl bromide is tolerated. Moreover, with the most reactive heteroarenes, this arylation could be performed in the "green" and renewable solvents 3-methylbutan-1-ol, pentan-1-ol or DEC. The use of a phosphine-free heterogeneous catalyst for such reactions reduces wastes and simplify purification procedure, and as the major by-products of these couplings are $\mathrm{KBr} / \mathrm{AcOH}$, this process is environmentally and economically attractive.

\section{Experimental Section}

Typical experiment for coupling reactions: The reaction of the aryl bromide $(1 \mathrm{mmol})$, heteroaromatic $(1.5 \mathrm{mmol})$ and KOAc $(0.196,2 \mathrm{mmol})$ in the presence of $10 \% \mathrm{Pd} / \mathrm{C}(0.011 \mathrm{~g}, 1 \mathrm{~mol} \%)$ in the appropriate solvent $\left(5 \mathrm{~mL}\right.$ ) (see schemes) under argon at $150{ }^{\circ} \mathrm{C}$ during $16 \mathrm{~h}$, affords the corresponding product after cooling, evaporation of the solvent and filtration on silica gel (pentane/ether).

4-(5-Pentylthiophen-2-yl)benzonitrile (1) ${ }^{[28]} \quad$ 4-Bromobenzonitrile $(0.182 \mathrm{~g}, 1 \mathrm{mmol})$ and 2-pentylthiophene $(0.231 \mathrm{~g}, 1.5 \mathrm{mmol})$ affords 1 in $91 \%(0.232 \mathrm{~g})$ yield as a colourless oil. ${ }^{1} \mathrm{H} \mathrm{NMR}\left(400 \mathrm{MHz}, \mathrm{CDCl}_{3}\right): \delta$ 7.65-7.57 (m, 4H), $7.23(\mathrm{~d}, J=3.6 \mathrm{~Hz}, 1 \mathrm{H}), 6.78(\mathrm{~d}, J=3.6 \mathrm{~Hz}, 1 \mathrm{H}), 2.83$ (t, $J=7.5 \mathrm{~Hz}, 2 \mathrm{H}), 1.78-1.66(\mathrm{~m}, 2 \mathrm{H}), 1.42-1.34(\mathrm{~m}, 4 \mathrm{H}), 0.92(\mathrm{t}, J=7.5$ $\mathrm{Hz}, 3 \mathrm{H}) .{ }^{13} \mathrm{C} \operatorname{NMR}\left(100 \mathrm{MHz}, \mathrm{CDCl}_{3}\right): \delta 148.4,139.2,138.9,132.6$, 125.6, 125.4, 124.8, 118.9, 109.8, 31.2, 30.2, 22.3, 13.9 .

\section{1-(4-(5-Pentylthiophen-2-yl)phenyl)ethan-1-one}

4Bromoacetophenone $(0.199 \mathrm{~g}, 1 \mathrm{mmol})$ and 2-pentylthiophene $(0.231 \mathrm{~g}$ $1.5 \mathrm{mmol})$ affords 2 in $91 \%(0.247 \mathrm{~g})$ yield as a yellow solid: $\mathrm{mp} 94-96^{\circ} \mathrm{C}$. ${ }^{1} \mathrm{H}$ NMR (400 MHz, $\left.\mathrm{CDCl}_{3}\right): \delta 7.93(\mathrm{~d}, J=8.5 \mathrm{~Hz}, 2 \mathrm{H}), 7.62(\mathrm{~d}, J=8.5 \mathrm{~Hz}$, $2 \mathrm{H}), 7.25(\mathrm{~d}, J=3.6 \mathrm{~Hz}, 1 \mathrm{H}), 6.78(\mathrm{~d}, J=3.6 \mathrm{~Hz}, 1 \mathrm{H}), 2.82(\mathrm{t}, J=7.5 \mathrm{~Hz}$, $2 \mathrm{H}), 2.59(\mathrm{~s}, 3 \mathrm{H}), 1.78-1.66(\mathrm{~m}, 2 \mathrm{H}), 1.41-1.33(\mathrm{~m}, 4 \mathrm{H}), 0.92(\mathrm{t}, J=7.5$ $\mathrm{Hz}, 3 \mathrm{H}) .{ }^{13} \mathrm{C}$ NMR $\left(100 \mathrm{MHz}, \mathrm{CDCl}_{3}\right): \delta 197.2,147.7,140.0,139.1$, 135.2, 129.0, 125.4, 125.0, 124.3, 31.2, 30.2, 26.4, 22.3, 13.9. elemental analysis: calcd (\%) for $\mathrm{C}_{17} \mathrm{H}_{20} \mathrm{OS}$ (272.41): C 74.96, $\mathrm{H} 7.40$; found: $\mathrm{C}$ 74.90, H 7.60 .

Ethyl 4-(5-pentylthiophen-2-yl)benzoate (3) Ethyl 4-bromobenzoate $(0.229 \mathrm{~g}, 1 \mathrm{mmol})$ and 2-pentylthiophene $(0.231 \mathrm{~g}, 1.5 \mathrm{mmol})$ affords 3 in $88 \%(0.266 \mathrm{~g})$ yield as a brown oil. ${ }^{1} \mathrm{H} \mathrm{NMR}\left(400 \mathrm{MHz}, \mathrm{CDCl}_{3}\right): \delta 8.02(\mathrm{~d}$, $J=8.5 \mathrm{~Hz}, 2 \mathrm{H}), 7.61(\mathrm{~d}, J=8.5 \mathrm{~Hz}, 2 \mathrm{H}), 7.22(\mathrm{~d}, J=3.6 \mathrm{~Hz}, 1 \mathrm{H}), 6.76(\mathrm{~d}$, $J=3.6 \mathrm{~Hz}, 1 \mathrm{H}), 4.38(\mathrm{q}, J=7.6 \mathrm{~Hz}, 2 \mathrm{H}), 2.81(\mathrm{t}, J=7.5 \mathrm{~Hz}, 2 \mathrm{H}), 1.78-$ $1.66(\mathrm{~m}, 2 \mathrm{H}), 1.47-1.34(\mathrm{~m}, 7 \mathrm{H}), 0.94(\mathrm{t}, J=7.5 \mathrm{~Hz}, 3 \mathrm{H}) .{ }^{13} \mathrm{C}$ NMR $(100$ $\left.\mathrm{MHz}, \mathrm{CDCl}_{3}\right): \delta 166.1,147.3,140.2,138.8,130.0,128.4,125.2,124.7$, $124.1,60.7,31.1,30.1,22.3,14.2,13.9$. elemental analysis: calcd (\%) for $\mathrm{C}_{18} \mathrm{H}_{22} \mathrm{O}_{2} \mathrm{~S}$ (302.43): C 71.49, $\mathrm{H} 7.33$; found: C 71.40, H 6.98.

2-(4-Nitrophenyl)-5-pentylthiophene (4) 4-Bromonitrobenzene $(0.202$ $\mathrm{g}, 1 \mathrm{mmol})$ and 2-pentylthiophene $(0.231 \mathrm{~g}, 1.5 \mathrm{mmol})$ affords $4 \mathrm{in} 67 \%$ $(0.184 \mathrm{~g})$ yield as a yellow solid: $\mathrm{mp} 60-62{ }^{\circ} \mathrm{C}$. ${ }^{1} \mathrm{H} \mathrm{NMR}(400 \mathrm{MHz}$, $\left.\mathrm{CDCl}_{3}\right): \delta 8.20(\mathrm{~d}, J=8.7 \mathrm{~Hz}, 2 \mathrm{H}), 7.66(\mathrm{~d}, J=8.7 \mathrm{~Hz}, 2 \mathrm{H}), 7.29(\mathrm{~d}, J=$ $3.6 \mathrm{~Hz}, 1 \mathrm{H}), 6.81(\mathrm{~d}, J=3.6 \mathrm{~Hz}, 1 \mathrm{H}), 2.84(\mathrm{t}, J=7.5 \mathrm{~Hz}, 2 \mathrm{H}), 1.77-1.66$ $(\mathrm{m}, 2 \mathrm{H}), 1.44-1.32(\mathrm{~m}, 4 \mathrm{H}), 0.93(\mathrm{t}, J=7.5 \mathrm{~Hz}, 3 \mathrm{H}) .{ }^{13} \mathrm{C} \mathrm{NMR}(100 \mathrm{MHz}$, 
$\left.\mathrm{CDCl}_{3}\right): \delta 149.3,146.2,141.0,138.8,125.9,125.6,125.4,124.4,31.2$, $30.3,22.4,14.0$. elemental analysis: calcd (\%) for $\mathrm{C}_{15} \mathrm{H}_{17} \mathrm{NO}_{2} \mathrm{~S}(275.37)$ : C 65.43, H 6.22; found: C 65.34, H 6.12.

2-Pentyl-5-(p-tolyl)thiophene (5) 4-Bromotoluene $(0.171 \mathrm{~g}, 1 \mathrm{mmol})$ and 2-pentylthiophene $(0.231 \mathrm{~g}, 1.5 \mathrm{mmol})$ affords 5 in $90 \%(0.220 \mathrm{~g})$

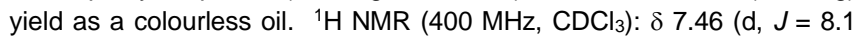
$\mathrm{Hz}, 2 \mathrm{H}), 7.17$ (d, $J=8.1 \mathrm{~Hz}, 2 \mathrm{H}), 7.08(\mathrm{~d}, J=3.6 \mathrm{~Hz}, 1 \mathrm{H}), 6.73(\mathrm{~d}, J=3.6$ $\mathrm{Hz}, 1 \mathrm{H}), 2.82(\mathrm{t}, J=7.5 \mathrm{~Hz}, 2 \mathrm{H}), 2.36(\mathrm{~s}, 3 \mathrm{H}), 1.78-1.65(\mathrm{~m}, 2 \mathrm{H}), 1.43$ $1.33(\mathrm{~m}, 4 \mathrm{H}), 0.92(\mathrm{t}, J=7.5 \mathrm{~Hz}, 3 \mathrm{H}) .{ }^{13} \mathrm{C}$ NMR $\left(100 \mathrm{MHz}, \mathrm{CDCl}_{3}\right): \delta$ 145.1, 141.7, 136.7, 132.0, 129.4, 125.4, 124.8, 122.1, 31.4, 31.3, 30.2, 22.4, 21.1, 14.0. elemental analysis: calcd (\%) for $\mathrm{C}_{16} \mathrm{H}_{20} \mathrm{~S}(244.40)$ : C 78.63, H 8.25; found: C 78.90, H 8.02

2-(4-Methoxyphenyl)-5-pentylthiophene (6) ${ }^{[29]}$ 4-Bromoanisole $(0.187$ $\mathrm{g}, 1 \mathrm{mmol})$ and 2-pentylthiophene $(0.231 \mathrm{~g}, 1.5 \mathrm{mmol})$ affords 6 in $76 \%$ $(0.198 \mathrm{~g})$ yield as a white solid: $\mathrm{mp} 62-64{ }^{\circ} \mathrm{C}$. ${ }^{1} \mathrm{H} \mathrm{NMR}(400 \mathrm{MHz}$, $\left.\mathrm{CDCl}_{3}\right): \delta 7.49(\mathrm{~d}, J=8.4 \mathrm{~Hz}, 2 \mathrm{H}), 7.02(\mathrm{~d}, J=3.6 \mathrm{~Hz}, 1 \mathrm{H}), 6.90(\mathrm{~d}, J=$ $8.4 \mathrm{~Hz}, 2 \mathrm{H}), 6.73(\mathrm{~d}, J=3.6 \mathrm{~Hz}, 1 \mathrm{H}), 3.83(\mathrm{~s}, 3 \mathrm{H}), 2.82(\mathrm{t}, J=7.5 \mathrm{~Hz}, 2 \mathrm{H}$ ) 1.77-1.66 (m, 2H), 1.44-1.32 (m, 4H), $0.93(\mathrm{t}, J=7.5 \mathrm{~Hz}, 3 \mathrm{H}) .{ }^{13} \mathrm{C}$ NMR $\left(100 \mathrm{MHz}, \mathrm{CDCl}_{3}\right): \delta 159.5,145.4,142.2,128.4,127.4,125.5,122.3$, $114.9,56.0,32.0,30.9,23.1,14.7$.

2-(5-Pentylthiophen-2-yl)benzonitrile (7) 2-Bromobenzonitrile $(0.182 \mathrm{~g}$ $1 \mathrm{mmol})$ and 2-pentylthiophene $(0.231 \mathrm{~g}, 1.5 \mathrm{mmol})$ affords 7 in $93 \%$ $(0.237 \mathrm{~g})$ yield as a colourless oil. ${ }^{1} \mathrm{H} \mathrm{NMR}\left(400 \mathrm{MHz}, \mathrm{CDCl}_{3}\right): \delta 7.71(\mathrm{dd}$ $J=8.0,0.8 \mathrm{~Hz}, 1 \mathrm{H}), 7.65-7.53(\mathrm{~m}, 2 \mathrm{H}), 7.51(\mathrm{~d}, J=3.6 \mathrm{~Hz}, 1 \mathrm{H}), 7.33(\mathrm{td}$, $J=8.0,1.7 \mathrm{~Hz}, 1 \mathrm{H}), 6.85(\mathrm{~d}, J=3.6 \mathrm{~Hz}, 1 \mathrm{H}), 2.86(\mathrm{t}, J=7.5 \mathrm{~Hz}, 2 \mathrm{H})$, 1.79-1.67 (m, 2H), 1.47-1.34 (m, 4H), $0.94(\mathrm{t}, J=7.5 \mathrm{~Hz}, 3 \mathrm{H}) .{ }^{13} \mathrm{C} N M R$ $\left(100 \mathrm{MHz}, \mathrm{CDCl}_{3}\right): \delta 148.4,137.7,136.5,134.2,132.7,129.1,127.3$, 126.8, 125.2, 119.0, 109.2, 31.2, 31.1, 30.0, 22.3, 13.9. elemental analysis: calcd (\%) for $\mathrm{C}_{16} \mathrm{H}_{17} \mathrm{NS}$ (255.38): C 75.25, $\mathrm{H} 6.71$; found: C 75.08, H 6.98

5-(4-Cyanophenyl)thiophene-2-carbonitrile (8) ${ }^{[17]}$ 4-Bromobenzonitrile (0.182 $\mathrm{g}, 1 \mathrm{mmol})$ and 2-thiophenecarbonitrile $(0.164 \mathrm{~g}, 1.5 \mathrm{mmol})$ affords 8 in $87 \%(0.183 \mathrm{~g})$ yield as a yellow solid: $\mathrm{mp} 212-214{ }^{\circ} \mathrm{C} .{ }^{1} \mathrm{H}$ NMR (400 MHz, $\left.\mathrm{CDCl}_{3}\right): \delta 7.73(\mathrm{~d}, J=8.5 \mathrm{~Hz}, 2 \mathrm{H}), 7.70(\mathrm{~d}, J=8.5 \mathrm{~Hz}$, 2H), 7.63 (d, J=3.9 Hz, 1H), 7.39 (d, J=3.9 Hz, 1H)

\section{5-(4-Acetylphenyl)thiophene-2-carbonitrile}

$(9)^{[17]}$ 4Bromoacetophenone $(0.199 \mathrm{~g}, 1 \mathrm{mmol})$ and 2-thiophenecarbonitrile $(0.164 \mathrm{~g}, 1.5 \mathrm{mmol})$ affords 9 in $90 \%(0.204 \mathrm{~g})$ yield as a yellow solid: $\mathrm{mp}$ $162-164{ }^{\circ} \mathrm{C} .{ }^{1} \mathrm{H}$ NMR $\left(400 \mathrm{MHz}, \mathrm{CDCl}_{3}\right): \delta 8.01(\mathrm{~d}, J=8.5 \mathrm{~Hz}, 2 \mathrm{H}), 7.69$ $7.93(\mathrm{~d}, J=8.5 \mathrm{~Hz}, 2 \mathrm{H}), 7.62(\mathrm{~d}, J=3.9 \mathrm{~Hz}, 1 \mathrm{H}), 7.39(\mathrm{~d}, J=3.9 \mathrm{~Hz}, 1 \mathrm{H})$, $2.62(\mathrm{~s}, 3 \mathrm{H})$

4-(5-Acetylthiophen-2-yl)benzonitrile (10) ${ }^{[17]} \quad$ 4-Bromobenzonitrile $(0.182 \mathrm{~g}, 1 \mathrm{mmol})$ and 2-acetylthiophene $(0.189 \mathrm{~g}, 1.5 \mathrm{mmol})$ affords 10 in $79 \%(0.179 \mathrm{~g})$ yield as a yellow solid: $\mathrm{mp} 166-168{ }^{\circ} \mathrm{C} .{ }^{1} \mathrm{H}$ NMR $(400$ $\left.\mathrm{MHz}, \mathrm{CDCl}_{3}\right): \delta 7.72(\mathrm{~d}, J=8.5 \mathrm{~Hz}, 2 \mathrm{H}), 7.70-7.65(\mathrm{~m}, 3 \mathrm{H}), 7.40(\mathrm{~d}, J=$ $3.9 \mathrm{~Hz}, 1 \mathrm{H}), 2.57$ (s, 3H).

Ethyl 5-(4-cyanophenyl)thiophene-2-carboxylate (11) ${ }^{[8 d]} \quad 4$ Bromobenzonitrile $(0.182 \mathrm{~g}, 1 \mathrm{mmol})$ and ethyl thiophene-2-carboxylate $(0.234 \mathrm{~g}, 1.5 \mathrm{mmol})$ affords 11 in $84 \%(0.216 \mathrm{~g})$ yield as a yellow solid: mp 134-136 ${ }^{\circ} \mathrm{C} .{ }^{1} \mathrm{H}$ NMR $\left(400 \mathrm{MHz}, \mathrm{CDCl}_{3}\right): \delta 7.77(\mathrm{~d}, J=3.9 \mathrm{~Hz}, 1 \mathrm{H})$, $7.72(\mathrm{~d}, J=8.5 \mathrm{~Hz}, 2 \mathrm{H}), 7.68(\mathrm{~d}, J=8.5 \mathrm{~Hz}, 2 \mathrm{H}), 7.38(\mathrm{~d}, J=3.9 \mathrm{~Hz}, 1 \mathrm{H})$, 4.37 (q, $J=7.6 \mathrm{~Hz}, 2 \mathrm{H}), 1.39(\mathrm{t}, J=7.6 \mathrm{~Hz}, 3 \mathrm{H})$.

3-(5-Pentylthiophen-2-yl)pyridine (12) 3-Brompyridine (0.158 g, 1 $\mathrm{mmol}$ ) and 2-pentylthiophene $(0.231 \mathrm{~g}, 1.5 \mathrm{mmol})$ affords 12 in $90 \%$ $(0.208 \mathrm{~g})$ yield as a colourless oil. ${ }^{1} \mathrm{H}$ NMR $\left(400 \mathrm{MHz}, \mathrm{CDCl}_{3}\right): \delta 8.81(\mathrm{~d}$,
$J=2.0 \mathrm{~Hz}, 1 \mathrm{H}$ ), $8.44(\mathrm{dd}, J=4.8,1.4 \mathrm{~Hz}, 1 \mathrm{H}), 7.77$ (ddd, $J=8.0,2.2,1.7$ $\mathrm{Hz}, 1 \mathrm{H}$ ), 7.22 (ddd, $J=8.0,4.8,0.6 \mathrm{~Hz}, 1 \mathrm{H}), 7.14(\mathrm{~d}, J=3.6 \mathrm{~Hz}, 1 \mathrm{H})$, $6.75(\mathrm{~d}, J=3.6 \mathrm{~Hz}, 1 \mathrm{H}), 2.80(\mathrm{t}, J=7.5 \mathrm{~Hz}, 2 \mathrm{H}), 1.75-1.63(\mathrm{~m}, 2 \mathrm{H}), 1.40-$ $1.28(\mathrm{~m}, 4 \mathrm{H}), 0.90(\mathrm{t}, J=7.5 \mathrm{~Hz}, 3 \mathrm{H}) .{ }^{13} \mathrm{C} \mathrm{NMR}\left(100 \mathrm{MHz}, \mathrm{CDCl}_{3}\right): \delta$ 147.9, 147.1, 146.6, 137.6, 132.4, 130.7, 125.3, 123.9, 123.5, 31.3, 31.2, $30.2,22.4,14.0$. elemental analysis: calcd (\%) for $\mathrm{C}_{14} \mathrm{H}_{17} \mathrm{NS}(231.36)$ : C 72.68, H 7.41; found: C 72.78, H 7.57.

4-(5-Butylfuran-2-yl)benzonitrile (13) ${ }^{[6 \mathrm{c}]}$ 4-Bromobenzonitrile $(0.182 \mathrm{~g}$, $1 \mathrm{mmol})$ and 2-butylfuran $(0.186 \mathrm{~g}, 1.5 \mathrm{mmol})$ affords 13 in $71 \%(0.160 \mathrm{~g})$ yield as a yellow solid: $\mathrm{mp} 58-60^{\circ} \mathrm{C}$. ${ }^{1} \mathrm{H} \mathrm{NMR}\left(400 \mathrm{MHz}, \mathrm{CDCl}_{3}\right): \delta 7.68$ $(\mathrm{d}, J=8.6 \mathrm{~Hz}, 2 \mathrm{H}), 7.61(\mathrm{~d}, J=8.6 \mathrm{~Hz}, 2 \mathrm{H}), 6.71(\mathrm{~d}, J=3.3 \mathrm{~Hz}, 1 \mathrm{H}), 6.11$ (d, $J=3.3 \mathrm{~Hz}, 1 \mathrm{H}$ ), 2.70 (t, $J=7.5 \mathrm{~Hz}, 2 \mathrm{H}), 1.67$ (quint., $J=7.5 \mathrm{~Hz}, 2 \mathrm{H}$ ), 1.43 (sext., $J=7.5 \mathrm{~Hz}, 2 \mathrm{H}), 0.96$ (t, $J=7.5 \mathrm{~Hz}, 3 \mathrm{H}) .{ }^{13} \mathrm{C} \mathrm{NMR}(100 \mathrm{MHz}$, $\left.\mathrm{CDCl}_{3}\right): \delta 158.5,150.1,134.9,132.5,123.3,119.1,109.4,109.1,107.6$, $30.0,27.9,22.2,13.4$

1-(4-(5-Butylfuran-2-yl)phenyl)ethan-1-one $\quad$ (14) $^{[15 a]}$ Bromoacetophenone $(0.199 \mathrm{~g}, 1 \mathrm{mmol})$ and 2-butylfuran $(0.186 \mathrm{~g}, 1.5$ $\mathrm{mmol}$ ) affords $14 \mathrm{in} 82 \%(0.198 \mathrm{~g})$ yield as a white solid: $\mathrm{mp} 43-45^{\circ} \mathrm{C}$. ${ }^{1} \mathrm{H}$ NMR $\left(400 \mathrm{MHz}, \mathrm{CDCl}_{3}\right): \delta 7.94(\mathrm{~d}, J=8.6 \mathrm{~Hz}, 2 \mathrm{H}), 7.68(\mathrm{~d}, J=8.6 \mathrm{~Hz}$, 2H), $6.70(\mathrm{~d}, J=3.3 \mathrm{~Hz}, 1 \mathrm{H}), 6.10(\mathrm{~d}, J=3.3 \mathrm{~Hz}, 1 \mathrm{H}), 2.70(\mathrm{t}, J=7.5 \mathrm{~Hz}$, 2H), 2.59 (s, 3H), 1.67 (quint., $J=7.5 \mathrm{~Hz}, 2 \mathrm{H}$ ), 1.43 (sext., $J=7.5 \mathrm{~Hz}$, $2 \mathrm{H}), 0.96(\mathrm{t}, J=7.5 \mathrm{~Hz}, 3 \mathrm{H}) .{ }^{13} \mathrm{C} \mathrm{NMR}\left(100 \mathrm{MHz} \mathrm{CDCl}_{3}\right): \delta 197.3,158.0$, 150.9, 135.2, 134.9, 128.9, 122.9, 108.4, 107.4, 30.1, 27.9, 26.4, 22.2.

2-Butyl-5-(p-tolyl)furan (15) ${ }^{[6 a]} \quad 4-B r o m o t o l u e n e ~(0.171 \mathrm{~g}, 1 \mathrm{mmol})$ and 2-butylfuran (0.186 g, $1.5 \mathrm{mmol}$ ) affords 15 in $67 \%(0.143 \mathrm{~g})$ yield as a yellow oil. ${ }^{1} \mathrm{H}$ NMR $\left(400 \mathrm{MHz}, \mathrm{CDCl}_{3}\right): \delta 7.53(\mathrm{~d}, J=8.1 \mathrm{~Hz}, 2 \mathrm{H}), 7.17(\mathrm{~d}$, $J=8.1 \mathrm{~Hz}, 2 \mathrm{H}), 6.48(\mathrm{~d}, J=3.3 \mathrm{~Hz}, 1 \mathrm{H}), 6.04(\mathrm{~d}, J=3.3 \mathrm{~Hz}, 1 \mathrm{H}), 2.70(\mathrm{t}$, $J=7.5 \mathrm{~Hz}, 2 \mathrm{H}$ ), 2.36 (s, 3H), 1.67 (quint., $J=7.5 \mathrm{~Hz}, 2 \mathrm{H}$ ), 1.43 (sext., $J=$ $7.5 \mathrm{~Hz}, 2 \mathrm{H}), 0.96$ (t, $J=7.5 \mathrm{~Hz}, 3 \mathrm{H})$.

2-(5-Butylfuran-2-yl)benzonitrile (16) ${ }^{[6 \mathrm{c}]}$ 2-Bromobenzonitrile $(0.182 \mathrm{~g}$, $1 \mathrm{mmol}$ ) and 2-butylfuran (0.186 g, $1.5 \mathrm{mmol})$ affords 16 in $88 \%(0.198 \mathrm{~g})$ yield as a yellow oil. ${ }^{1} \mathrm{H}$ NMR $\left(400 \mathrm{MHz}, \mathrm{CDCl}_{3}\right): \delta 7.82(\mathrm{~d}, J=8.6 \mathrm{~Hz}$, $1 \mathrm{H}), 7.64(\mathrm{~d}, J=8.6 \mathrm{~Hz}, 1 \mathrm{H}), 7.55$ (td, $J=7.8,1.3 \mathrm{~Hz}, 1 \mathrm{H}), 7.24$ (td, $J=$ 7.8, 1.1 Hz, 1H), $7.20(\mathrm{~d}, J=3.3 \mathrm{~Hz}, 1 \mathrm{H}), 6.15(\mathrm{~d}, J=3.3 \mathrm{~Hz}, 1 \mathrm{H}), 2.71(\mathrm{t}$, $J=7.5 \mathrm{~Hz}, 2 \mathrm{H}$ ), 1.67 (quint., $J=7.5 \mathrm{~Hz}, 2 \mathrm{H}$ ), 1.43 (sext., $J=7.5 \mathrm{~Hz}, 2 \mathrm{H}$ ), $0.96(\mathrm{t}, J=7.5 \mathrm{~Hz}, 3 \mathrm{H}) .{ }^{13} \mathrm{C} \operatorname{NMR}\left(100 \mathrm{MHz} \mathrm{CDCl}_{3}\right): \delta 157.9,147.9$, 134.0, 133.4, 132.7, 126.3, 125.3, 119.1, 111.3, 107.6, 106.0, 29.9, 27.7, 22.2, 13.7 .

3-(5-Butylfuran-2-yl)pyridine (17) ${ }^{[6 \mathrm{c}]} \quad$ 3-Bromopyridine $(0.158 \mathrm{~g}, 1$ mmol) and 2-butylfuran (0.186 g, $1.5 \mathrm{mmol})$ affords 17 in $79 \%(0.159 \mathrm{~g})$ yield as a yellow oil. ${ }^{1} \mathrm{H}$ NMR $\left(400 \mathrm{MHz}, \mathrm{CDCl}_{3}\right): \delta 8.87(\mathrm{~s}, 1 \mathrm{H}), 8.42(\mathrm{~d}$, $J=4.8 \mathrm{~Hz}, 1 \mathrm{H}), 7.86(\mathrm{dd}, J=8.0,2.2 \mathrm{~Hz}, 1 \mathrm{H}), 7.24(\mathrm{dd}, J=8.0,4.8 \mathrm{~Hz}$, $1 \mathrm{H}), 6.61(\mathrm{~d}, J=3.3 \mathrm{~Hz}, 1 \mathrm{H}), 6.07(\mathrm{~d}, J=3.3 \mathrm{~Hz}, 1 \mathrm{H}), 2.68$ (t, $J=7.5 \mathrm{~Hz}$, 2H), 1.67 (quint., $J=7.5 \mathrm{~Hz}, 2 \mathrm{H}$ ), 1.40 (sext., $J=7.5 \mathrm{~Hz}, 2 \mathrm{H}$ ), 0.94 (t, $J=$ $7.5 \mathrm{~Hz}, 3 \mathrm{H})$.

4-(2-Ethylbenzofuran-3-yl)benzonitrile (18) ${ }^{[6 \mathrm{~d}]} \quad$ 4-Bromobenzonitrile $(0.182 \mathrm{~g}, 1 \mathrm{mmol})$ and 2-ethylbenzofuran $(0.219 \mathrm{~g}, 1.5 \mathrm{mmol})$ affords 18 in $83 \%(0.205 \mathrm{~g})$ yield as a white solid: $\mathrm{mp} 114-116{ }^{\circ} \mathrm{C} .{ }^{1} \mathrm{H}$ NMR $(400$ $\left.\mathrm{MHz}_{\mathrm{CDCl}}\right): \delta 7.77(\mathrm{~d}, J=8.6 \mathrm{~Hz}, 2 \mathrm{H}), 7.60(\mathrm{~d}, J=8.6 \mathrm{~Hz}, 2 \mathrm{H}), 7.53(\mathrm{~d}$ $J=8.2 \mathrm{~Hz}, 1 \mathrm{H}), 7.50(\mathrm{~d}, J=8.2 \mathrm{~Hz}, 1 \mathrm{H}), 7.32(\mathrm{t}, J=8.0 \mathrm{~Hz}, 1 \mathrm{H}), 7.26(\mathrm{t}$, $J=8.0 \mathrm{~Hz}, 1 \mathrm{H}), 2.90(\mathrm{q}, J=7.5 \mathrm{~Hz}, 2 \mathrm{H}), 1.38(\mathrm{t}, J=7.5 \mathrm{~Hz}, 3 \mathrm{H}) .{ }^{13} \mathrm{C}$ NMR $\left(100 \mathrm{MHz}_{\mathrm{C}} \mathrm{CDCl}_{3}\right): \delta 157.2,154.0,138.0,132.5,129.4,127.8$, $124.1,123.0,119.0,118.8,114.9,111.1,110.5,20.3,12.8$.

4-(1-Methylpyrrol-2-yl)benzonitrile (19) $)^{[7 c]}$ 4-Bromobenzonitrile $(0.182$ $\mathrm{g}, 1 \mathrm{mmol})$ and $\mathrm{N}$-methylpyrrole $(0.324 \mathrm{~g}, 4 \mathrm{mmol})$ affords 19 in $84 \%$ $(0.153 \mathrm{~g})$ yield as a white solid: $\mathrm{mp} 102-104{ }^{\circ} \mathrm{C} .{ }^{1} \mathrm{H} \mathrm{NMR}(400 \mathrm{MHz}$, 
$\left.\mathrm{CDCl}_{3}\right): \delta 7.70(\mathrm{~d}, J=8.4 \mathrm{~Hz}, 2 \mathrm{H}), 7.53(\mathrm{~d}, J=8.4 \mathrm{~Hz}, 2 \mathrm{H}), 6.82(\mathrm{t}, J=$ $2.2 \mathrm{~Hz}, 1 \mathrm{H}), 6.38$ (dd, $J=3.6,1.7 \mathrm{~Hz}, 1 \mathrm{H}), 6.27$ (dd, $J=3.6,2.6 \mathrm{~Hz}, 1 \mathrm{H}$ ), $3.75(\mathrm{~s}, 3 \mathrm{H})$

Ethyl 4-(1-methylpyrrol-2-yl)benzoate (20) ${ }^{[30]}$ Ethyl 4-bromobenzoate $(0.229 \mathrm{~g}, 1 \mathrm{mmol})$ and $\mathrm{N}$-methylpyrrole $(0.324 \mathrm{~g}, 4 \mathrm{mmol})$ affords 20 in $76 \%(0.174 \mathrm{~g})$ yield as a yellow solid: $\mathrm{mp} 61-63^{\circ} \mathrm{C}$. ${ }^{1} \mathrm{H} \mathrm{NMR}(400 \mathrm{MHz}$, $\left.\mathrm{CDCl}_{3}\right): \delta 8.11(\mathrm{~d}, J=8.5 \mathrm{~Hz}, 2 \mathrm{H}), 7.51(\mathrm{~d}, J=8.5 \mathrm{~Hz}, 2 \mathrm{H}), 6.79(\mathrm{t}, J=$ $2.2 \mathrm{~Hz}, 1 \mathrm{H}), 6.38(\mathrm{dd}, J=3.6,1.7 \mathrm{~Hz}, 1 \mathrm{H}), 6.26(\mathrm{dd}, J=3.6,2.6 \mathrm{~Hz}, 1 \mathrm{H})$, $4.43(\mathrm{q}, J=7.6 \mathrm{~Hz}, 2 \mathrm{H}), 3.74(\mathrm{~s}, 3 \mathrm{H}), 1.45(\mathrm{t}, J=7.6 \mathrm{~Hz}, 3 \mathrm{H})$.

1-Methyl-2-(p-tolyl)pyrrole (21) ${ }^{[7 c]}$ 4-Bromotoluene $(0.171 \mathrm{~g}, 1 \mathrm{mmol})$ and $\mathrm{N}$-methylpyrrole $(0.324 \mathrm{~g}, 4 \mathrm{mmol})$ affords 21 in $52 \%(0.089 \mathrm{~g})$ yield as a yellow oil. ${ }^{1} \mathrm{H}$ NMR $\left(400 \mathrm{MHz}, \mathrm{CDCl}_{3}\right): \delta 7.30(\mathrm{~d}, J=8.3 \mathrm{~Hz}, 2 \mathrm{H})$, $7.21(\mathrm{~d}, J=8.3 \mathrm{~Hz}, 2 \mathrm{H}), 6.70(\mathrm{t}, J=2.2 \mathrm{~Hz}, 1 \mathrm{H}), 6.22-6.18(\mathrm{~m}, 2 \mathrm{H}), 3.65$ $(\mathrm{s}, 3 \mathrm{H}), 2.38(\mathrm{~s}, 3 \mathrm{H})$

2-(1-Methylpyrrol-2-yl)benzonitrile (22) ${ }^{[7 c]}$ 2-Bromobenzonitrile $(0.182$ $\mathrm{g}, 1 \mathrm{mmol})$ and $\mathrm{N}$-methylpyrrole $(0.324 \mathrm{~g}, 4 \mathrm{mmol})$ affords 22 in $87 \%$ $(0.158 \mathrm{~g})$ yield as a white solid: $\mathrm{mp} 94-96{ }^{\circ} \mathrm{C}$. ${ }^{1} \mathrm{H} \mathrm{NMR}(400 \mathrm{MHz}$, $\left.\mathrm{CDCl}_{3}\right): \delta 7.74(\mathrm{~d}, J=7.8 \mathrm{~Hz}, 1 \mathrm{H}), 7.61(\mathrm{td}, J=7.8,1.4 \mathrm{~Hz}, 1 \mathrm{H}), 7.44(\mathrm{~d}$, $J=7.8 \mathrm{~Hz}, 1 \mathrm{H}$ ), 7.40 (td, $J=8.5,1.2 \mathrm{~Hz}, 1 \mathrm{H}), 6.80(\mathrm{t}, J=2.2 \mathrm{~Hz}, 1 \mathrm{H}$ ), 6.42 (dd, $J=3.6,1.7 \mathrm{~Hz}, 1 \mathrm{H}), 6.26$ (dd, $J=3.6,2.6 \mathrm{~Hz}, 1 \mathrm{H}), 3.62(\mathrm{~s}, 3 \mathrm{H})$.

\section{4-(2-Isopropyl-4-methylthiazol-5-yl)benzonitrile}

(23)

$4-$

Bromobenzonitrile $(0.182 \mathrm{~g}, 1 \mathrm{mmol})$ and 2-isopropyl-4-methylthiazole $(0.212 \mathrm{~g}, 1.5 \mathrm{mmol})$ affords 23 in $97 \%(0.234 \mathrm{~g})$ yield as a white solid: $\mathrm{mp}$ 58-60 ${ }^{\circ} \mathrm{C}$. ${ }^{1} \mathrm{H}$ NMR $\left(400 \mathrm{MHz}, \mathrm{CDCl}_{3}\right): \delta 7.70(\mathrm{~d}, J=8.5 \mathrm{~Hz}, 2 \mathrm{H}), 7.53(\mathrm{~d}$, $J=8.5 \mathrm{~Hz}, 2 \mathrm{H}$ ), 3.30 (sept., $J=7.5 \mathrm{~Hz}, 1 \mathrm{H}$ ), 2.50 (s, 3H), 1.42 (d, $J=7.5$ $\mathrm{Hz}, 6 \mathrm{H}) .{ }^{13} \mathrm{C}$ NMR $\left(100 \mathrm{MHz}, \mathrm{CDCl}_{3}\right): \delta 176.8,148.5,137.4,132.3$, $129.4,128.6,118.5,110.8,33.4,23.1,16.4$. elemental analysis: calcd (\%) for $\mathrm{C}_{14} \mathrm{H}_{14} \mathrm{~N}_{2} \mathrm{~S}$ (242.34): C 69.39, H 5.82; found: C 69.14, H 5.78.

\section{4-(2-Isopropyl-4-methylthiazol-5-yl)benzaldehyde}

(24)

$4-$

Bromobenzaldehyde $(0.185 \mathrm{~g}, 1 \mathrm{mmol})$ and 2-isopropyl-4-methylthiazole $(0.212 \mathrm{~g}, 1.5 \mathrm{mmol})$ affords 24 in $94 \%(0.230 \mathrm{~g})$ yield as a yellow oil. ${ }^{1} \mathrm{H}$ NMR (400 MHz, CDCl $)$ ): $\delta 9.98(\mathrm{~s}, 1 \mathrm{H}), 7.86(\mathrm{~d}, J=8.5 \mathrm{~Hz}, 2 \mathrm{H}), 7.54(\mathrm{~d}$, $J=8.5 \mathrm{~Hz}, 2 \mathrm{H}$ ), 3.25 (sept., $J=7.5 \mathrm{~Hz}, 1 \mathrm{H}), 2.48(\mathrm{~s}, 3 \mathrm{H}), 1.38(\mathrm{~d}, J=7.5$ $\mathrm{Hz}, 6 \mathrm{H}) .{ }^{13} \mathrm{C} \mathrm{NMR}\left(100 \mathrm{MHz} \mathrm{CDCl}_{3}\right): \delta 191.3,176.5,148.3,138.8$, 134.9, 129.9, 129.2, 129.1, 33.3, 23.0, 16.4. elemental analysis: calcd (\%) for $\mathrm{C}_{14} \mathrm{H}_{15} \mathrm{NOS}$ (245.34): C 68.54, H 6.16; found: C 68.30, H 6.01.

1-(4-(2-Isopropyl-4-methylthiazol-5-yl)phenyl)ethan-1-one (25) 4 Bromoacetophenone $(0.199 \mathrm{~g}, 1 \mathrm{mmol})$ and 2-isopropyl-4-methylthiazole $(0.212 \mathrm{~g}, 1.5 \mathrm{mmol})$ affords $25 \mathrm{in} 97 \%(0.251 \mathrm{~g})$ yield as a yellow oil. ${ }^{1} \mathrm{H}$ NMR (400 MHz, $\left.\mathrm{CDCl}_{3}\right): \delta 7.96(\mathrm{~d}, J=8.0 \mathrm{~Hz}, 2 \mathrm{H}), 7.49(\mathrm{~d}, J=8.0 \mathrm{~Hz}$ 2H), 3.27 (sept., $J=7.5 \mathrm{~Hz}, 1 \mathrm{H}$ ), 2.59 (s, 3H), 2.48 (s, 3H), 1.39 (d, $J=$ $7.5 \mathrm{~Hz}, 6 \mathrm{H}) .{ }^{13} \mathrm{C} \mathrm{NMR}\left(100 \mathrm{MHz}, \mathrm{CDCl}_{3}\right): \delta 197.2,176.3,148.0,137.4$, 135.7, 129.3, 128.9, 128.6, 33.3, 26.5, 23.1, 16.4. elemental analysis: calcd (\%) for $\mathrm{C}_{14} \mathrm{H}_{17} \mathrm{NOS}$ (259.37): C 69.46, H 6.61; found: C 69.38, H 6.40 .

Ethyl 4-(2-isopropyl-4-methylthiazol-5-yl)benzoate (26) Ethyl 4bromobenzoate $(0.229 \mathrm{~g}, 1 \mathrm{mmol})$ and 2-isopropyl-4-methylthiazole $(0.212 \mathrm{~g}, 1.5 \mathrm{mmol})$ affords 26 in $95 \%(0.274 \mathrm{~g})$ yield as a yellow oil. ${ }^{1} \mathrm{H}$ NMR (400 MHz, $\left.\mathrm{CDCl}_{3}\right): \delta 8.05(\mathrm{~d}, J=8.5 \mathrm{~Hz}, 2 \mathrm{H}), 7.47(\mathrm{~d}, J=8.5 \mathrm{~Hz}$, 2H), 4.37 (q, $J=7.5 \mathrm{~Hz}, 2 \mathrm{H}$ ), 3.25 (sept., $J=7.5 \mathrm{~Hz}, 1 \mathrm{H}$ ), 2.48 (s, 3H), 1.43-1.35 (m, 9H). ${ }^{13} \mathrm{C}$ NMR $\left(100 \mathrm{MHz}, \mathrm{CDCl}_{3}\right): \delta 176.0,166.0,147.8$, 137.1, 129.7, 129.4, 129.1, 128.7, 60.9, 33.3, 23.0, 16.3, 14.2. elemental analysis: calcd (\%) for $\mathrm{C}_{16} \mathrm{H}_{19} \mathrm{NO}_{2} \mathrm{~S}$ (289.39): C 66.41, $\mathrm{H} \mathrm{6.62}$; found: C $66.58, \mathrm{H} 6.45$
2-Isopropyl-4-methyl-5-(4-nitrophenyl)thiazole

(27)

4Bromonitrobenzene $(0.202 \mathrm{~g}, 1 \mathrm{mmol})$ and 2-isopropyl-4-methylthiazole $(0.212 \mathrm{~g}, 1.5 \mathrm{mmol})$ affords $27 \mathrm{in} 96 \%(0.251 \mathrm{~g})$ yield as a yellow oil. ${ }^{1} \mathrm{H}$ NMR (400 MHz, CDCl $): \delta 8.24(\mathrm{~d}, J=8.5 \mathrm{~Hz}, 2 \mathrm{H}), 7.57(\mathrm{~d}, J=8.5 \mathrm{~Hz}$, 2H), 3.28 (sept., $J=7.5 \mathrm{~Hz}, 1 \mathrm{H}$ ), 2.51 (s, 3H), 1.42 (d, $J=7.5 \mathrm{~Hz}, 6 \mathrm{H}$ ). ${ }^{13} \mathrm{C}$ NMR $\left(100 \mathrm{MHz}, \mathrm{CDCl}_{3}\right): \delta 177.0,148.9,146.5,139.4,129.3,128.2$, 123.8, 33.3, 23.0, 16.5. elemental analysis: calcd (\%) for $\mathrm{C}_{13} \mathrm{H}_{14} \mathrm{~N}_{2} \mathrm{O}_{2} \mathrm{~S}$ (262.33): C 59.52, H 5.38; found: C 59.60, H 5.49.

5-(4-Fluorophenyl)-2-isopropyl-4-methylthiazole

(28)

4-

Fluorobromobenzene $(0.175 \mathrm{~g}, 1 \mathrm{mmol})$ and 2-isopropyl-4-methylthiazole $(0.211 \mathrm{~g}, 1.5 \mathrm{mmol})$ affords $28 \mathrm{in} 97 \%(0.228 \mathrm{~g})$ yield as a yellow oil. ${ }^{1} \mathrm{H}$ $\operatorname{NMR}\left(400 \mathrm{MHz}, \mathrm{CDCl}_{3}\right): \delta 7.36(\mathrm{dd}, J=8.6,5.3 \mathrm{~Hz}, 2 \mathrm{H}), 7.07(\mathrm{t}, J=8.6$ $\mathrm{Hz}, 2 \mathrm{H}$ ), 3.27 (sept., $J=7.5 \mathrm{~Hz}, 1 \mathrm{H}$ ), 2.42 (s, 3H), 1.40 (d, $J=7.5 \mathrm{~Hz}$, $6 \mathrm{H}) .{ }^{13} \mathrm{C}$ NMR $\left(100 \mathrm{MHz}, \mathrm{CDCl}_{3}\right): \delta 175.3,162.1(\mathrm{~d}, J=247.8 \mathrm{~Hz}), 146.9$, 130.8 (d, $J=8.1 \mathrm{~Hz}), 129.3,128.5$ (d, $J=3.5 \mathrm{~Hz}), 115.6(\mathrm{~d}, J=21.8 \mathrm{~Hz}$ ), 33.3, 23.2, 15.9. elemental analysis: calcd (\%) for $\mathrm{C}_{13} \mathrm{H}_{14} \mathrm{FNS}(235.32)$ : C 66.35, H 6.00; found: C 66.20, H 5.74.

2-Isopropyl-4-methyl-5-(p-tolyl)thiazole (29) 4-Bromotoluene (0.171 g, $1 \mathrm{mmol})$ and 2-isopropyl-4-methylthiazole $(0.211 \mathrm{~g}, 1.5 \mathrm{mmol})$ affords 29 in $92 \%(0.212 \mathrm{~g})$ yield as a yellow oil. ${ }^{1} \mathrm{H} \mathrm{NMR}\left(400 \mathrm{MHz}, \mathrm{CDCl}_{3}\right): \delta 7.31$ $(\mathrm{d}, J=8.1 \mathrm{~Hz}, 2 \mathrm{H}), 7.20$ (d, $J=8.1 \mathrm{~Hz}, 2 \mathrm{H}), 3.27$ (sept., $J=7.5 \mathrm{~Hz}, 1 \mathrm{H}$ ), $2.46(\mathrm{~s}, 3 \mathrm{H}), 2.38(\mathrm{~s}, 3 \mathrm{H}), 1.41(\mathrm{~d}, J=7.5 \mathrm{~Hz}, 6 \mathrm{H}) .{ }^{13} \mathrm{C} \mathrm{NMR}(100 \mathrm{MHz}$ $\left.\mathrm{CDCl}_{3}\right): \delta 174.8,146.4,137.2,130.4,129.5,129.2,128.9,33.3,23.2$ 21.1, 16.0. elemental analysis: calcd (\%) for $\mathrm{C}_{14} \mathrm{H}_{17} \mathrm{NS}(231.36)$ : $\mathrm{C} 72.68$, $\mathrm{H} 7.41$; found: C 72.64, H 7.32 .

5-(4-(tert-Butyl)phenyl)-2-isopropyl-4-methylthiazole $\quad(\mathbf{3 0}) \quad$ 4-tert Butylbromobenzene $(0.213 \mathrm{~g}, 1 \mathrm{mmol})$ and 2-isopropyl-4-methylthiazole $(0.211 \mathrm{~g}, 1.5 \mathrm{mmol})$ affords $30 \mathrm{in} 89 \%(0.243 \mathrm{~g})$ yield as a colourless oil. ${ }^{1} \mathrm{H}$ NMR $\left(400 \mathrm{MHz}_{\mathrm{CDCl}}\right.$ ): $\delta 7.45(\mathrm{~d}, J=8.0 \mathrm{~Hz}, 2 \mathrm{H}), 7.38(\mathrm{~d}, J=8.1 \mathrm{~Hz}$, 2H), 3.28 (sept., $J=7.5 \mathrm{~Hz}, 1 \mathrm{H}), 2.47$ (s, 3H), 1.41 (d, $J=7.5 \mathrm{~Hz}, 6 \mathrm{H})$, 1.35 (s, 9H). ${ }^{13} \mathrm{C} \mathrm{NMR}\left(100 \mathrm{MHz}, \mathrm{CDCl}_{3}\right): \delta 174.9,150.4,146.5,130.4$, 129.6, 128.8, 125.5, 34.6, 33.3, 31.2, 23.2, 16.1. elemental analysis: calcd (\%) for $\mathrm{C}_{17} \mathrm{H}_{23} \mathrm{NS}$ (273.44): C 74.67, H 8.48; found: C 74.81, H 8.31.

2-Isopropyl-5-(4-methoxyphenyl)-4-methylthiazole (31) 4Bromoanisole $(0.187 \mathrm{~g}, 1 \mathrm{mmol})$ and 2-isopropyl-4-methylthiazole $(0.211$ $\mathrm{g}, 1.5 \mathrm{mmol})$ affords 31 in $72 \%(0.178 \mathrm{~g})$ yield as a colourless oil. ${ }^{1} \mathrm{H}$ NMR $\left(400 \mathrm{MHz}, \mathrm{CDCl}_{3}\right): \delta 7.33(\mathrm{~d}, J=8.0 \mathrm{~Hz}, 2 \mathrm{H}), 6.93(\mathrm{~d}, J=8.0 \mathrm{~Hz}$ 2H), 3.84 (s, 3H), 3.25 (sept., $J=7.5 \mathrm{~Hz}, 1 \mathrm{H}), 2.43(\mathrm{~s}, 3 \mathrm{H}), 1.40$ (d, $J=$ $7.5 \mathrm{~Hz}, 6 \mathrm{H}) .{ }^{13} \mathrm{C}$ NMR $\left(100 \mathrm{MHz}, \mathrm{CDCl}_{3}\right): \delta 174.7,159.0,146.2,130.4$, $130.2,124.8,114.0,55.3,33.3,23.2,16.0$. elemental analysis: calcd (\%) for $\mathrm{C}_{14} \mathrm{H}_{17} \mathrm{NOS}$ (247.36): C 67.98, H 6.93; found: C 68.12, H 6.78.

4-(2-Isopropyl-4-methylthiazol-5-yl)- $N, N$-dimethylaniline (32) Bromo- $N, N$-dimethylaniline $(0.200 \mathrm{~g}, 1 \mathrm{mmol})$ and 2-isopropyl-4methylthiazole $(0.211 \mathrm{~g}, 1.5 \mathrm{mmol})$ affords $32 \mathrm{in} 78 \%(0.203 \mathrm{~g})$ yield as a yellow oil. ${ }^{1} \mathrm{H}$ NMR $\left(400 \mathrm{MHz}, \mathrm{CDCl}_{3}\right): \delta 7.29(\mathrm{~d}, J=8.3 \mathrm{~Hz}, 2 \mathrm{H}), 6.74(\mathrm{~d}$, $J=8.3 \mathrm{~Hz}, 2 \mathrm{H}$ ), 3.27 (sept., $J=7.5 \mathrm{~Hz}, 1 \mathrm{H}), 2.99(\mathrm{~s}, 6 \mathrm{H}), 2.45$ (s, 3H), $1.40(\mathrm{~d}, J=7.5 \mathrm{~Hz}, 6 \mathrm{H}) .{ }^{13} \mathrm{C}$ NMR $\left(100 \mathrm{MHz}, \mathrm{CDCl}_{3}\right): \delta 174.0,149.8$, $145.5,131.1,130.0,120.2,112.2,40.4,33.3,23.3,16.1$. elemental analysis: calcd (\%) for $\mathrm{C}_{15} \mathrm{H}_{20} \mathrm{~N}_{2} \mathrm{~S}$ (260.40): C 69.19, H 7.74; found: C $69.00, \mathrm{H} 7.88$.

5-(3,5-Bis(trifluoromethyl)phenyl)-2-isopropyl-4-methylthiazole (33) 3,5-Bis(trifluoromethyl)bromobenzene $(0.293 \mathrm{~g}, 1 \mathrm{mmol})$ and 2-isopropyl4-methylthiazole $(0.211 \mathrm{~g}, 1.5 \mathrm{mmol})$ affords 33 in $96 \%(0.339 \mathrm{~g})$ yield as a colourless oil. ${ }^{1} \mathrm{H}$ NMR $\left(400 \mathrm{MHz} \mathrm{CDCl}_{3}\right): \delta 7.87(\mathrm{~s}, 2 \mathrm{H}), 7.85(\mathrm{~s}, 1 \mathrm{H})$ 3.33 (sept., $J=7.5 \mathrm{~Hz}, 1 \mathrm{H}), 2.51(\mathrm{~s}, 3 \mathrm{H}), 1.45$ (d, $J=7.5 \mathrm{~Hz}, 6 \mathrm{H}) .{ }^{13} \mathrm{C}$ NMR $\left(100 \mathrm{MHz}_{\mathrm{CDCl}}\right): \delta 177.1,148.9,135.0,132.0(\mathrm{q}, J=33.5 \mathrm{~Hz}$ ) $129.0(\mathrm{~m}), 127.3,123.1(\mathrm{q}, J=273.0 \mathrm{~Hz}), 121.0(\mathrm{~m}), 33.5,23.1,16.1$ 
elemental analysis: calcd (\%) for $\mathrm{C}_{15} \mathrm{H}_{13} \mathrm{~F}_{6} \mathrm{NS}$ (353.33): C 50.99, H 3.71; found: C 51.20, H 3.64 .

\section{2-(2-Isopropyl-4-methylthiazol-5-yl)benzonitrile \\ (34) \\ Bromobenzonitrile $(0.182 \mathrm{~g}, 1 \mathrm{mmol})$ and 2-isopropyl-4-methylthiazole $(0.212 \mathrm{~g}, 1.5 \mathrm{mmol})$ affords 34 in $94 \%(0.227 \mathrm{~g})$ yield as a colourless oil. ${ }^{1} \mathrm{H}$ NMR $\left(400 \mathrm{MHz}, \mathrm{CDCl}_{3}\right): \delta 7.73(\mathrm{dd}, J=7.8,1.4 \mathrm{~Hz}, 1 \mathrm{H}), 7.60$ (td, $J=$ 7.8, $1.4 \mathrm{~Hz}, 1 \mathrm{H}$ ), 7.48-7.42 (m, 2H), 3.28 (sept., $J=7.5 \mathrm{~Hz}, 1 \mathrm{H}$ ), 2.34 (s, $3 \mathrm{H}), 1.40(\mathrm{~d}, J=7.5 \mathrm{~Hz}, 6 \mathrm{H}) .{ }^{13} \mathrm{C}$ NMR $\left(100 \mathrm{MHz}, \mathrm{CDCl}_{3}\right): \delta 177.2$, 149.9, 136.1, 133.3, 132.5, 131.8, 128.3, 125.5, 117.7, 113.5, 33.3, 23.0, 16.0. elemental analysis: calcd (\%) for $\mathrm{C}_{14} \mathrm{H}_{14} \mathrm{~N}_{2} \mathrm{~S}$ (242.34): C 69.39, $\mathrm{H}$ 5.82; found: C 69.50 , H 5.98 .}

\section{2-Isopropyl-4-methyl-5-(4-nitrophenyl)thiazole}

Bromonitrobenzene $(0.202 \mathrm{~g}, 1 \mathrm{mmol})$ and 2-isopropyl-4-methylthiazole $(0.212 \mathrm{~g}, 1.5 \mathrm{mmol})$ affords $35 \mathrm{in} 66 \%(0.173 \mathrm{~g})$ yield as a yellow oil. ${ }^{1} \mathrm{H}$ $\operatorname{NMR}\left(400 \mathrm{MHz}, \mathrm{CDCl}_{3}\right): \delta 7.94(\mathrm{dd}, J=8.1,1.2 \mathrm{~Hz}, 1 \mathrm{H}), 7.61(\mathrm{td}, J=7.5$ $1.3 \mathrm{~Hz}, 1 \mathrm{H}), 7.54(\mathrm{td}, J=7.5,1.3 \mathrm{~Hz}, 1 \mathrm{H}), 7.44(\mathrm{dd}, J=8.0,1.3 \mathrm{~Hz}, 1 \mathrm{H})$, 3.29 (sept., $J=7.5 \mathrm{~Hz}, 1 \mathrm{H}), 2.21(\mathrm{~s}, 3 \mathrm{H}), 1.41$ (d, $J=7.5 \mathrm{~Hz}, 6 \mathrm{H}) .{ }^{13} \mathrm{C}$ NMR $\left(100 \mathrm{MHz}, \mathrm{CDCl}_{3}\right): \delta 177.1,150.0,149.5,133.6,132.4,129.3$, 126.9, 124.4, 123.9, 33.3, 23.1, 15.5. elemental analysis: calcd (\%) for $\mathrm{C}_{13} \mathrm{H}_{14} \mathrm{~N}_{2} \mathrm{O}_{2} \mathrm{~S}$ (262.33): C 59.52, H 5.38; found: C 59.47, H 5.20

2-Isopropyl-4-methyl-5-(2-(trifluoromethyl)phenyl)thiazole (36) 2(Trifluoromethyl)bromobenzene $(0.225 \mathrm{~g}, 1 \mathrm{mmol})$ and 2-isopropyl-4 methylthiazole $(0.212 \mathrm{~g}, 1.5 \mathrm{mmol})$ affords 36 in $87 \%(0.248 \mathrm{~g})$ yield as a colourless oil. ${ }^{1} \mathrm{H}$ NMR $\left(400 \mathrm{MHz}, \mathrm{CDCl}_{3}\right): \delta 7.72(\mathrm{~d}, J=7.5 \mathrm{~Hz}, 1 \mathrm{H})$, $7.52(\mathrm{t}, J=7.5 \mathrm{~Hz}, 1 \mathrm{H}), 746(\mathrm{td}, J=7.5 \mathrm{~Hz}, 1 \mathrm{H}), 7.34(\mathrm{~d}, J=7.6 \mathrm{~Hz}, 1 \mathrm{H})$, 3.27 (sept., $J=7.5 \mathrm{~Hz}, 1 \mathrm{H}$ ), $2.14(\mathrm{~s}, 3 \mathrm{H}), 1.39$ (d, $J=7.5 \mathrm{~Hz}, 6 \mathrm{H}$ ). ${ }^{13} \mathrm{C}$ NMR $\left(100 \mathrm{MHz}, \mathrm{CDCl}_{3}\right): \delta 176.5,149.6,133.7,131.4,131.1,130.6$ (q, $=29.8 \mathrm{~Hz}$ ), 128.7, 126.2 (q, $J=5.3 \mathrm{~Hz}), 125.4,123.6$ (q, $J=273.8 \mathrm{~Hz})$, 33.2, 23.1, 15.5. elemental analysis: calcd (\%) for $\mathrm{C}_{14} \mathrm{H}_{14} \mathrm{~F}_{3} \mathrm{NS}$ (285.33): C 58.93, H 4.95; found: C 58.99, H 4.20.

\section{5-(2-Fluorophenyl)-2-isopropyl-4-methylthiazole}

(37)

Fluorobromobenzene $(0.175 \mathrm{~g}, 1 \mathrm{mmol})$ and 2-isopropyl-4-methylthiazole $(0.211 \mathrm{~g}, 1.5 \mathrm{mmol})$ affords 37 in $93 \%(0.218 \mathrm{~g})$ yield as a colourless oil. ${ }^{1} \mathrm{H}$ NMR $\left(400 \mathrm{MHz}, \mathrm{CDCl}_{3}\right): \delta$ 7.38-7.30 (m, 2H), 7.20-7.12 (m, 2H), 3.31 (sept., $J=7.5 \mathrm{~Hz}, 1 \mathrm{H}), 2.36(\mathrm{~s}, 3 \mathrm{H}), 1.42(\mathrm{~d}, J=7.5 \mathrm{~Hz}, 6 \mathrm{H}) .{ }^{13} \mathrm{C}$ NMR $\left(100 \mathrm{MHz}, \mathrm{CDCl}_{3}\right): \delta 176.9,159.7(\mathrm{~d}, J=248.8 \mathrm{~Hz}$ ), 149.2, 132.1 (d, $J=$ $2.6 \mathrm{~Hz}), 129.8(\mathrm{~d}, J=8.1 \mathrm{~Hz}), 124.1(\mathrm{~d}, J=3.8 \mathrm{~Hz}), 123.0,120.0(\mathrm{~d}, J=$ $15.3 \mathrm{~Hz}$ ), $116.0(\mathrm{~d}, J=22.2 \mathrm{~Hz}), 33.3,23.2,15.9(\mathrm{~d}, J=2.8 \mathrm{~Hz})$. elemental analysis: calcd (\%) for $\mathrm{C}_{13} \mathrm{H}_{14} \mathrm{FNS}$ (235.32): C 66.35, H 6.00; found: C $66.54, \mathrm{H} 5.78$.

2-Isopropyl-4-methyl-5-(pyridin-3-yl)thiazole (38) 3-Bromopyridine $(0.158 \mathrm{~g}, 1 \mathrm{mmol})$ and 2-isopropyl-4-methylthiazole $(0.211 \mathrm{~g}, 1.5 \mathrm{mmol})$ affords 38 in $95 \%(0.207 \mathrm{~g})$ yield as a colourless oil. ${ }^{1} \mathrm{H} \mathrm{NMR}(400 \mathrm{MHz}$, $\left.\mathrm{CDCl}_{3}\right): \delta 8.68(\mathrm{bs}, 1 \mathrm{H}), 8.55$ (bs, $\left.1 \mathrm{H}\right), 7.70(\mathrm{dd}, J=7.9,1.5 \mathrm{~Hz}, 1 \mathrm{H}), 7.32$ (dd, $J=7.7,5.0 \mathrm{~Hz}, 1 \mathrm{H}$ ), 3.25 (sept., $J=7.5 \mathrm{~Hz}, 1 \mathrm{H}$ ), 2.45 (s, 3H), 1.40 $(\mathrm{d}, J=7.5 \mathrm{~Hz}, 6 \mathrm{H}) .{ }^{13} \mathrm{C}$ NMR $\left(100 \mathrm{MHz}, \mathrm{CDCl}_{3}\right): \delta 176.4,146.6,148.4$, 148.2, 136.1, 128.8, 126.5, 123.3, 33.4, 23.1, 16.0. elemental analysis: calcd (\%) for $\mathrm{C}_{12} \mathrm{H}_{14} \mathrm{~N}_{2} \mathrm{~S}$ (218.32): C 66.02, H 6.46; found: C 65.87, H 6.34 .

2-Isopropyl-4-methyl-5-(quinolin-3-yl)thiazole (39) 3-Bromoquinoline $(0.208 \mathrm{~g}, 1 \mathrm{mmol})$ and 2-isopropyl-4-methylthiazole $(0.211 \mathrm{~g}, 1.5 \mathrm{mmol})$ affords 39 in $96 \%(0.257 \mathrm{~g})$ yield as a yellow oil. ${ }^{1} \mathrm{H}$ NMR $(400 \mathrm{MHz}$, $\left.\mathrm{CDCl}_{3}\right): \delta 8.99(\mathrm{~d}, J=1.9 \mathrm{~Hz}, 1 \mathrm{H}), 8.15(\mathrm{~d}, J=1.9 \mathrm{~Hz}, 1 \mathrm{H}), 8.12(\mathrm{~d}, J=$ $8.1 \mathrm{~Hz}, 1 \mathrm{H}), 7.82(\mathrm{~d}, J=8.1 \mathrm{~Hz}, 1 \mathrm{H}), 7.72(\mathrm{t}, J=8.0 \mathrm{~Hz}, 1 \mathrm{H}), 7.57(\mathrm{t}, J=$ $8.0 \mathrm{~Hz}, 1 \mathrm{H}$ ), 3.30 (sept., $J=7.5 \mathrm{~Hz}, 1 \mathrm{H}$ ), 2.54 (s, $3 \mathrm{H}$ ), 1.44 (d, $J=7.5 \mathrm{~Hz}$, $6 \mathrm{H}) .{ }^{13} \mathrm{C}$ NMR $\left(100 \mathrm{MHz}, \mathrm{CDCl}_{3}\right): \delta 176.4,150.6,148.4,147.0,135.1$ $129.6,129.2,127.7,127.5,127.2,126.7,125.9,33.4,23.1,16.1$ elemental analysis: calcd (\%) for $\mathrm{C}_{16} \mathrm{H}_{16} \mathrm{~N}_{2} \mathrm{~S}$ (268.38): C 71.61, $\mathrm{H} 6.01$ found: C 71.42, H 5.88.

\section{4-(2-Ethyl-4-methylthiazol-5-yl)benzonitrile $\quad(40)^{[8 a]}$} Bromobenzonitrile $(0.182 \mathrm{~g}, 1 \mathrm{mmol})$ and 2-ethyl-4-methylthiazole $(0.190$ $\mathrm{g}, 1.5 \mathrm{mmol})$ affords 40 in $96 \%(0.219 \mathrm{~g})$ yield as a white solid: $\mathrm{mp} 54$ $56{ }^{\circ} \mathrm{C} .{ }^{1} \mathrm{H}$ NMR $\left(400 \mathrm{MHz}, \mathrm{CDCl}_{3}\right): \delta 7.71(\mathrm{~d}, J=8.5 \mathrm{~Hz}, 2 \mathrm{H}), 7.54(\mathrm{~d}, J$ $=8.5 \mathrm{~Hz}, 2 \mathrm{H}), 3.04(\mathrm{q}, J=7.5 \mathrm{~Hz}, 2 \mathrm{H}), 2.51(\mathrm{~s}, 3 \mathrm{H}), 1.43(\mathrm{t}, J=7.5 \mathrm{~Hz}$, $3 \mathrm{H})$

1-(4-(2-Ethyl-4-methylthiazol-5-yl)phenyl)propan-1-one (41) Bromopropiophenone $(0.213 \mathrm{~g}, 1 \mathrm{mmol})$ and 2-ethyl-4-methylthiazole $(0.190 \mathrm{~g}, 1.5 \mathrm{mmol})$ affords 41 in $96 \%(0.248 \mathrm{~g})$ yield as a colourless oil. ${ }^{1} \mathrm{H} \mathrm{NMR}\left(400 \mathrm{MHz}, \mathrm{CDCl}_{3}\right): \delta 7.97(\mathrm{~d}, J=8.5 \mathrm{~Hz}, 2 \mathrm{H}), 7.48(\mathrm{~d}, J=8.5 \mathrm{~Hz}$, 2H), 2.98 (q, $J=7.5 \mathrm{~Hz}, 4 \mathrm{H}), 2.47(\mathrm{~s}, 3 \mathrm{H}), 1.38(\mathrm{t}, J=7.5 \mathrm{~Hz}, 3 \mathrm{H}), 1.22$ (t, $J=7.5 \mathrm{~Hz}, 3 \mathrm{H}) .{ }^{13} \mathrm{C} \mathrm{NMR}\left(100 \mathrm{MHz}, \mathrm{CDCl}_{3}\right): \delta 199.9,171.1,148.0$, $137.0,135.5,129.8,128.9,128.3,31.7,26.9,16.3,14.2,8.2$. elemental analysis: calcd (\%) for $\mathrm{C}_{15} \mathrm{H}_{17} \mathrm{NOS}$ (259.37): C 69.46, H 6.61; found: C $69.60, \mathrm{H} 6.51$.

4-(1-Methylimidazol-5-yl)benzonitrile (42) ${ }^{[9 e]} \quad$ 4-Bromobenzonitrile $(0.182 \mathrm{~g}, 1 \mathrm{mmol})$ and 1 -methylimidazole $(0.123 \mathrm{~g}, 1.5 \mathrm{mmol})$ affords 42 in $84 \%(0.154 \mathrm{~g})$ yield as a white solid: $\mathrm{mp} 146-148{ }^{\circ} \mathrm{C}$. ${ }^{1} \mathrm{H}$ NMR $(400$ $\left.\mathrm{MHz}, \mathrm{CDCl}_{3}\right): \delta 7.70$ (d, $\left.J=8.4 \mathrm{~Hz}, 2 \mathrm{H}\right), 7.56$ (bs, $\left.1 \mathrm{H}\right), 7.50$ (d, $J=8.4 \mathrm{~Hz}$, $2 \mathrm{H}), 7.20$ (bs, $1 \mathrm{H}), 3.71(\mathrm{~s}, 3 \mathrm{H})$

1-Methyl-5-( $p$-tolyl)imidazole (43) $)^{[9]}$ 4-Bromotoluene $(0.171 \mathrm{~g}, 1 \mathrm{mmol})$ and 1-methylimidazole $(0.123 \mathrm{~g}, 1.5 \mathrm{mmol})$ affords 43 in $73 \%(0.125 \mathrm{~g})$ yield as a white solid: $\mathrm{mp} 40-42{ }^{\circ} \mathrm{C} .{ }^{1} \mathrm{H}$ NMR $\left(400 \mathrm{MHz}, \mathrm{CDCl}_{3}\right): \delta 7.31$ (d, $J=8.1 \mathrm{~Hz}, 2 \mathrm{H}$ ), 7.53 (bs, $1 \mathrm{H}), 7.26$ (d, $J=8.1 \mathrm{~Hz}, 2 \mathrm{H}$ ), 7.09 (bs, $1 \mathrm{H}$ ), $3.67(\mathrm{~s}, 3 \mathrm{H}), 2.42(\mathrm{~s}, 3 \mathrm{H})$

2-(1-Methylimidazol-5-yl)benzonitrile (44) 2-Bromobenzonitrile $(0.182$ $\mathrm{g}, 1 \mathrm{mmol})$ and 1 -methylimidazole $(0.123 \mathrm{~g}, 1.5 \mathrm{mmol})$ affords 44 in $82 \%$ $(0.150 \mathrm{~g})$ yield as a white solid: $\mathrm{mp} 156-158{ }^{\circ} \mathrm{C}$. ${ }^{1} \mathrm{H}$ NMR $(400 \mathrm{MHz}$ $\left.\mathrm{CDCl}_{3}\right): \delta 7.71(\mathrm{~d}, J=8.4 \mathrm{~Hz}, 1 \mathrm{H}), 7.61(\mathrm{td}, J=7.8,1.3 \mathrm{~Hz}, 1 \mathrm{H}), 7.52$ (bs, $1 \mathrm{H}), 7.44(\mathrm{td}, J=7.8,1.1 \mathrm{~Hz}, 1 \mathrm{H}), 7.38(\mathrm{~d}, J=8.0 \mathrm{~Hz}, 1 \mathrm{H}), 7.16(\mathrm{bs}, 1 \mathrm{H})$ $3.56(\mathrm{~s}, 3 \mathrm{H}) .{ }^{13} \mathrm{C} \mathrm{NMR}\left(100 \mathrm{MHz}, \mathrm{CDCl}_{3}\right): \delta 139.8,133.6,133.2,132.9$, $131.0,130.3,129.3,128.8,117.9,113.0,32.3$. elemental analysis: calcd (\%) for $\mathrm{C}_{11} \mathrm{H}_{9} \mathrm{~N}_{3}$ (183.21): C 72.11, H 4.95; found: C 72.00, H 4.87.

3-(1-Methylimidazol-5-yl)pyridine (45) $\left.{ }^{[9]}\right]$ 3-Bromopyridine $(0.158 \mathrm{~g}, 1$ $\mathrm{mmol})$ and 1-methylimidazole $(0.123 \mathrm{~g}, 1.5 \mathrm{mmol})$ affords 45 in $80 \%$ $(0.127 \mathrm{~g})$ yield as a white solid: $\mathrm{mp} 124-126{ }^{\circ} \mathrm{C} .{ }^{1} \mathrm{H}$ NMR $(400 \mathrm{MHz}$, $\left.\mathrm{CDCl}_{3}\right): \delta 8.70(\mathrm{~d}, J=1.6 \mathrm{~Hz}, 1 \mathrm{H}), 8.63(\mathrm{dd}, J=4.8,1.4 \mathrm{~Hz}, 1 \mathrm{H}), 7.72(\mathrm{dt}$ $J=8.0,1.9 \mathrm{~Hz}, 1 \mathrm{H}), 7.59$ (bs, $1 \mathrm{H}), 7.40$ (dd, $J=8.0,4.8 \mathrm{~Hz}, 1 \mathrm{H}), 7.19$ (bs, $1 \mathrm{H}), 3.71(\mathrm{~s}, 3 \mathrm{H})$

4-(3,5-Dimethylisoxazol-4-yl)benzonitrile (46) ${ }^{[10]}$ 4-Bromobenzonitrile $(0.182 \mathrm{~g}, 1 \mathrm{mmol})$ and 3,5-dimethylisoxazole $(0.144 \mathrm{~g}, 1.5 \mathrm{mmol})$ affords 46 in $93 \%(0.184 \mathrm{~g})$ yield as a white solid: $\mathrm{mp} 113-115^{\circ} \mathrm{C}$. ${ }^{1} \mathrm{H}$ NMR $(400$ $\left.\mathrm{MHz}, \mathrm{CDCl}_{3}\right): \delta 7.73(\mathrm{~d}, J=8.1 \mathrm{~Hz}, 2 \mathrm{H}), 7.38(\mathrm{~d}, J=8.1 \mathrm{~Hz}, 2 \mathrm{H}), 2.42(\mathrm{~s}$, $3 \mathrm{H}), 2.27(\mathrm{~s}, 3 \mathrm{H})$.

Ethyl 4-(3,5-dimethylisoxazol-4-yl)benzoate (47) $)^{[31]}$ Ethyl 4 bromobenzoate $(0.229 \mathrm{~g}, 1 \mathrm{mmol})$ and 3,5 -dimethylisoxazole $(0.144 \mathrm{~g}$, $1.5 \mathrm{mmol}$ ) affords 47 in $92 \%(0.225 \mathrm{~g})$ yield as a white solid: $\mathrm{mp} 68-70^{\circ} \mathrm{C}$. ${ }^{1} \mathrm{H} \mathrm{NMR}\left(400 \mathrm{MHz}, \mathrm{CDCl}_{3}\right): \delta 8.09(\mathrm{~d}, J=8.1 \mathrm{~Hz}, 2 \mathrm{H}), 7.32(\mathrm{~d}, J=8.1 \mathrm{~Hz}$, 2H), $4.38(\mathrm{q}, J=7.6 \mathrm{~Hz}, 2 \mathrm{H}), 2.40(\mathrm{~s}, 3 \mathrm{H}), 2.26(\mathrm{~s}, 3 \mathrm{H}), 1.38(\mathrm{t}, J=7.6$ $\mathrm{Hz}, 3 \mathrm{H}) .{ }^{13} \mathrm{C}$ NMR $\left(100 \mathrm{MHz}, \mathrm{CDCl}_{3}\right): \delta 166.0,165.6,158.2,135.1$, $129.9,129.5,128.8,115.9,61.0,14.2,11.5,10.7$ 
4-(4-Methoxyphenyl)-3,5-dimethylisoxazole (48) ${ }^{[10]}$ 4-Bromoanisole $(0.187 \mathrm{~g}, 1 \mathrm{mmol})$ and 3,5-dimethylisoxazole $(0.144 \mathrm{~g}, 1.5 \mathrm{mmol})$ affords 48 in $75 \%(0.152 \mathrm{~g})$ yield as a colourless oil. ${ }^{1} \mathrm{H}$ NMR $\left(400 \mathrm{MHz}, \mathrm{CDCl}_{3}\right)$ : $\delta 7.17(\mathrm{~d}, J=8.0 \mathrm{~Hz}, 2 \mathrm{H}), 6.96(\mathrm{~d}, J=8.0 \mathrm{~Hz}, 2 \mathrm{H}), 3.85(\mathrm{~s}, 3 \mathrm{H}), 2.38$ (s, $3 \mathrm{H}), 2.25(\mathrm{~s}, 3 \mathrm{H}) .{ }^{13} \mathrm{C}$ NMR $\left(100 \mathrm{MHz}, \mathrm{CDCl}_{3}\right): \delta 164.8,159.0,158.8$, $130.3,122.6,116.2,114.2,55.3,11.5,10.8$

2-(3,5-Dimethylisoxazol-4-yl)benzonitrile (49) ${ }^{[10]}$ 2-Bromobenzonitrile $(0.182 \mathrm{~g}, 1 \mathrm{mmol})$ and 3,5-dimethylisoxazole $(0.144 \mathrm{~g}, 1.5 \mathrm{mmol})$ affords 49 in $91 \%(0.180 \mathrm{~g})$ yield as a white solid: $\mathrm{mp} 115-117^{\circ} \mathrm{C} .{ }^{1} \mathrm{H}$ NMR (400 $\left.\mathrm{MHz}, \mathrm{CDCl}_{3}\right): \delta 7.76(\mathrm{~d}, J=7.8 \mathrm{~Hz}, 1 \mathrm{H}), 7.66(\mathrm{td}, J=7.9,1.4 \mathrm{~Hz}, 1 \mathrm{H})$, 7.49 (td, $J=8.5,1.2 \mathrm{~Hz}, 1 \mathrm{H}), 7.32$ (d, $J=7.8 \mathrm{~Hz}, 1 \mathrm{H}), 2.35$ (s, 3H), 2.19 $(\mathrm{s}, 3 \mathrm{H})$.

4-(Imidazo[1,2-a]pyridin-3-yl)benzonitrile (50) ${ }^{[9 f]}$ 4-Bromobenzonitrile $(0.182 \mathrm{~g}, 1 \mathrm{mmol})$ and imidazo[1,2-a]pyridine $(0.177 \mathrm{~g}, 1.5 \mathrm{mmol})$ affords 50 in $94 \%(0.206 \mathrm{~g})$ yield as a yellow solid: $\mathrm{mp} 175-177{ }^{\circ} \mathrm{C} .{ }^{1} \mathrm{H}$ NMR $\left(400 \mathrm{MHz}, \mathrm{CDCl}_{3}\right): \delta 8.38(\mathrm{~d}, J=6.8 \mathrm{~Hz}, 1 \mathrm{H}), 7.84-7.72(\mathrm{~m}, 4 \mathrm{H}), 7.69(\mathrm{~d}$, $J=8.2 \mathrm{~Hz}, 2 \mathrm{H}), 7.29$ (dd, $J=8.6,6.8 \mathrm{~Hz}, 1 \mathrm{H}), 6.92(\mathrm{t}, J=6.8 \mathrm{~Hz}, 1 \mathrm{H})$.

1-(4-(Imidazo[1,2-a]pyridin-3-yl)phenyl)ethan-1-one $\quad(51)^{[9 f]}$ Bromoacetophenone $(0.199 \mathrm{~g}, 1 \mathrm{mmol})$ and imidazo[1,2-a]pyridine $(0.177 \mathrm{~g}, 1.5 \mathrm{mmol})$ affords 51 in $96 \%(0.226 \mathrm{~g})$ yield as a yellow solid: $\mathrm{mp} 160-162{ }^{\circ} \mathrm{C} .{ }^{1} \mathrm{H}$ NMR $\left(400 \mathrm{MHz}, \mathrm{CDCl}_{3}\right): \delta 8.35(\mathrm{~d}, J=6.9 \mathrm{~Hz}, 1 \mathrm{H})$, $8.04(\mathrm{~d}, J=8.2 \mathrm{~Hz}, 2 \mathrm{H}), 7.73(\mathrm{~s}, 1 \mathrm{H}), 7.70-7.60(\mathrm{~m}, 3 \mathrm{H}), 7.19(\mathrm{t}, J=7.2$ $\mathrm{Hz}, 1 \mathrm{H}), 6.82(\mathrm{t}, J=7.2 \mathrm{~Hz}, 1 \mathrm{H}) .2 .59(\mathrm{~s}, 3 \mathrm{H})$.

3-(4-Methoxyphenyl)imidazo[1,2-a]pyridine (52) ${ }^{[9 f]} \quad$ 4-Bromoanisole $(0.187 \mathrm{~g}, 1 \mathrm{mmol})$ and imidazo[1,2-a]pyridine $(0.177 \mathrm{~g}, 1.5 \mathrm{mmol})$ affords 52 in $93 \%(0.208 \mathrm{~g})$ yield as a white solid: $\mathrm{mp} 85-87^{\circ} \mathrm{C} .{ }^{1} \mathrm{H}$ NMR $(400$ $\left.\mathrm{MHz}, \mathrm{CDCl}_{3}\right): \delta 8.24(\mathrm{~d}, J=6.8 \mathrm{~Hz}, 1 \mathrm{H}), 7.68-7.60(\mathrm{~m}, 2 \mathrm{H}), 7.45(\mathrm{~d}, J=$ $8.8 \mathrm{~Hz}, 2 \mathrm{H}), 7.17(\mathrm{t}, J=8.0 \mathrm{~Hz}, 1 \mathrm{H}), 7.03(\mathrm{~d}, J=8.0 \mathrm{~Hz}, 2 \mathrm{H}), 6.76(\mathrm{t}, J=$ $6.8 \mathrm{~Hz}, 1 \mathrm{H}), 3.85(\mathrm{~s}, 3 \mathrm{H})$.

2-(Imidazo[1,2-a]pyridin-3-yl)benzonitrile (53) ${ }^{[9 f]}$ 2-Bromobenzonitrile $(0.182 \mathrm{~g}, 1 \mathrm{mmol})$ and imidazo[1,2-a]pyridine $(0.177 \mathrm{~g}, 1.5 \mathrm{mmol})$ affords 53 in $92 \%(0.201 \mathrm{~g})$ yield as a white solid: $\mathrm{mp} 148-150{ }^{\circ} \mathrm{C}$. ${ }^{1} \mathrm{H}$ NMR $(400$ $\mathrm{MHz}$, acetone- $\left.d_{6}\right): \delta 8.35(\mathrm{~d}, J=6.8 \mathrm{~Hz}, 1 \mathrm{H}), 7.99(\mathrm{dd}, J=7.8,0.8 \mathrm{~Hz}$, $1 \mathrm{H}), 7.92-7.80(\mathrm{~m}, 3 \mathrm{H}), 7.71-7.62(\mathrm{~m}, 2 \mathrm{H}), 7.36$ (ddd, $J=8.6,6.8,1.1 \mathrm{~Hz}$ 1H), 6.99 (td, $J=6.8,0.9 \mathrm{~Hz}, 1 \mathrm{H})$

3-(Imidazo[1,2-a]pyridin-3-yl)quinoline $\quad \mathbf{( 5 4 )}^{[9 f]} \quad$ 3-Bromoquinoline $(0.208 \mathrm{~g}, 1 \mathrm{mmol})$ and imidazo[1,2-a]pyridine $(0.177 \mathrm{~g}, 1.5 \mathrm{mmol})$ affords 54 in $94 \%(0.230 \mathrm{~g})$ yield as a yellow solid: $\mathrm{mp} 146-148{ }^{\circ} \mathrm{C} .{ }^{1} \mathrm{H}$ NMR $\left(400 \mathrm{MHz}, \mathrm{CDCl}_{3}\right): \delta 9.12(\mathrm{~d}, J=2.1 \mathrm{~Hz}, 1 \mathrm{H}), 8.38(\mathrm{dt}, J=7.0,1.0 \mathrm{~Hz}$, $1 \mathrm{H}), 8.32(\mathrm{~d}, J=2.1 \mathrm{~Hz}, 1 \mathrm{H}), 8.17(\mathrm{~d}, J=8.2 \mathrm{~Hz}, 1 \mathrm{H}), 7.88(\mathrm{~d}, J=8.3 \mathrm{~Hz}$, $1 \mathrm{H}), 7.86(\mathrm{~s}, 1 \mathrm{H}), 7.80-7.70(\mathrm{~m}, 2 \mathrm{H}), 7.61(\mathrm{td}, \mathrm{J}=7.0,1.1,1 \mathrm{H}), 7.27$ (ddd, $J=8.6,6.8,1.1 \mathrm{~Hz}, 1 \mathrm{H}), 6.87(\mathrm{td}, J=6.8,0.9 \mathrm{~Hz}, 1 \mathrm{H})$.

4-(Imidazo[1,2-b]pyridazin-3-yl)benzonitrile $\quad(55)^{[15 b]}$ Bromobenzonitrile $(0.182 \mathrm{~g}, 1 \mathrm{mmol})$ and imidazo[1,2-b]pyridazine $(0.178$ $\mathrm{g}, 1.5 \mathrm{mmol})$ affords 55 in $95 \%(0.209 \mathrm{~g})$ yield as a white solid: $\mathrm{mp} 159$ $161^{\circ} \mathrm{C} .{ }^{1} \mathrm{H}$ NMR $\left(400 \mathrm{MHz}, \mathrm{CDCl}_{3}\right): \delta 8.44(\mathrm{~d}, J=4.3 \mathrm{~Hz}, 1 \mathrm{H}), 8.22(\mathrm{~d}, J$ $=8.5 \mathrm{~Hz}, 2 \mathrm{H}), 8.15(\mathrm{~s}, 1 \mathrm{H}), 8.03(\mathrm{~d}, J=9.0 \mathrm{~Hz}, 1 \mathrm{H}), 7.73(\mathrm{~d}, J=8.5 \mathrm{~Hz}$, 2H), 7.13 (dd, $J=9.0,4.3 \mathrm{~Hz}, 1 \mathrm{H})$.

\section{3-(4-Nitrophenyl)imidazo[1,2-b]pyridazine}

(56) ${ }^{[15 b]}$

Bromonitrobenzene $(0.202 \mathrm{~g}, 1 \mathrm{mmol})$ and imidazo[1,2-b]pyridazine $(0.178 \mathrm{~g}, 1.5 \mathrm{mmol})$ affords 56 in $96 \%(0.230 \mathrm{~g})$ yield as a yellow solid: mp 207-209 ${ }^{\circ} \mathrm{C}$. ${ }^{1} \mathrm{H}$ NMR $\left(400 \mathrm{MHz}\right.$, acetone- $\left.d_{6}\right): \delta 8.6(\mathrm{dd}, J=4.3,1.3$ $\mathrm{Hz}, 1 \mathrm{H}), 8.52(\mathrm{~d}, J=8.9 \mathrm{~Hz}, 2 \mathrm{H}), 8.42(\mathrm{~s}, 1 \mathrm{H}), 8.33(\mathrm{~d}, J=8.9 \mathrm{~Hz}, 2 \mathrm{H})$, 8.15 (dd, $J=8.2,1.6 \mathrm{~Hz}, 1 \mathrm{H}), 7.37$ (dd, $J=8.2,4.3 \mathrm{~Hz}, 1 \mathrm{H}$ ).
3-(4-Chlorophenyl)imidazo[1,2-b]pyridazine $\quad(57)^{[15 b]} \quad$ 4Bromochlorobenzene $(0.191 \mathrm{~g}, 1 \mathrm{mmol})$ and imidazo[1,2-b]pyridazine $(0.178 \mathrm{~g}, 1.5 \mathrm{mmol})$ affords 57 in $93 \%(0.213 \mathrm{~g})$ yield as a yellow solid: mp 144-146 ${ }^{\circ} \mathrm{C} .{ }^{1} \mathrm{H}$ NMR $\left(400 \mathrm{MHz}, \mathrm{CDCl}_{3}\right): \delta 8.47(\mathrm{~d}, J=4.0 \mathrm{~Hz}, 1 \mathrm{H})$, $8.13(\mathrm{~d}, J=9.0 \mathrm{~Hz}, 1 \mathrm{H}), 8.07(\mathrm{~s}, 1 \mathrm{H}), 8.00(\mathrm{~d}, J=8.5 \mathrm{~Hz}, 2 \mathrm{H}), 7.48(\mathrm{~d}, J$ $=8.5 \mathrm{~Hz}, 2 \mathrm{H}), 7.15(\mathrm{dd}, J=9.0,4.0 \mathrm{~Hz}, 1 \mathrm{H})$.

3-(4-Methoxyphenyl)imidazo[1,2-b]pyridazine (58) ${ }^{[15 b]}$ 4-Bromoanisole $(0.187 \mathrm{~g}, 1 \mathrm{mmol})$ and imidazo[1,2-b]pyridazine $(0.178 \mathrm{~g}, 1.5 \mathrm{mmol})$ affords 58 in $90 \%(0.202 \mathrm{~g})$ yield as a yellow solid: $\mathrm{mp} 76-78{ }^{\circ} \mathrm{C}$. ${ }^{1} \mathrm{H}$ NMR (400 MHz, $\mathrm{CDCl}_{3}$ ): $\delta 8.37$ (dd, $\left.J=4.4,1.4 \mathrm{~Hz}, 1 \mathrm{H}\right), 8.00$ (dd, $J=$ 9.1, $1.4 \mathrm{~Hz}, 1 \mathrm{H}), 7.98-7.92(\mathrm{~m}, 3 \mathrm{H}), 7.06-6.98(\mathrm{~m}, 3 \mathrm{H}), 3.85(\mathrm{~s}, 3 \mathrm{H})$.

2-(Imidazo[1,2-b]pyridazin-3-yl)benzonitrile $\quad(59)^{[15 b]}$ Bromobenzonitrile $(0.182 \mathrm{~g}, 1 \mathrm{mmol})$ and imidazo[1,2-b]pyridazine $(0.178$ $\mathrm{g}, 1.5 \mathrm{mmol})$ affords $59 \mathrm{in} 96 \%(0.211 \mathrm{~g})$ yield as a yellow solid: $\mathrm{mp} 214-$ $216{ }^{\circ} \mathrm{C}$. ${ }^{1} \mathrm{H}$ NMR $\left(400 \mathrm{MHz}, \mathrm{CDCl}_{3}\right): \delta 8.47$ (dd, $\left.J=4.2,1.0 \mathrm{~Hz}, 1 \mathrm{H}\right)$, $8.23(\mathrm{~s}, 1 \mathrm{H}), 8.18(\mathrm{dd}, J=9.2,1.3 \mathrm{~Hz}, 1 \mathrm{H}), 7.98(\mathrm{~d}, J=7.8 \mathrm{~Hz}, 1 \mathrm{H}), 7.86$ (dd, $J=7.8,1.0 \mathrm{~Hz}, 1 \mathrm{H}), 7.75$ (td, $J=7.8,1.3 \mathrm{~Hz}, 1 \mathrm{H}), 7.54$ (t, $J=7.7$, $1.0 \mathrm{~Hz}, 1 \mathrm{H}), 7.23(\mathrm{dd}, J=9.2,4.2 \mathrm{~Hz}, 1 \mathrm{H})$.

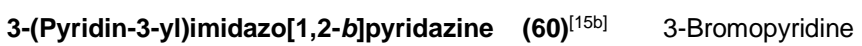
(0.158 $\mathrm{g}, 1 \mathrm{mmol})$ and imidazo[1,2-b]pyridazine (0.178 $\mathrm{g}, 1.5 \mathrm{mmol})$ affords 60 in $88 \%(0.172 \mathrm{~g})$ yield as a yellow solid: $\mathrm{mp} 112-114{ }^{\circ} \mathrm{C} .{ }^{1} \mathrm{H}$ NMR $\left(400 \mathrm{MHz}, \mathrm{CDCl}_{3}\right): \delta 9.23(\mathrm{~d}, J=1.8 \mathrm{~Hz}, 1 \mathrm{H}), 8.58(\mathrm{dd}, J=4.8,1.4$ $\mathrm{Hz}, 1 \mathrm{H}), 8.46-8.36(\mathrm{~m}, 2 \mathrm{H}), 8.10(\mathrm{~s}, 1 \mathrm{H}), 8.02(\mathrm{dd}, J=7.2,1.6 \mathrm{~Hz}, 1 \mathrm{H})$, 7.39 (dd, $J=8.0,4.8 \mathrm{~Hz}, 1 \mathrm{H}$ ), 7.09 (dd, $J=9.2,4.4 \mathrm{~Hz}, 1 \mathrm{H}$ ).

\section{Acknowledgments}

We thank CNRS and ANR for financial support.

Keywords: Palladium • Heterogeneous catalysis • Direct arylation $\cdot \mathrm{C}-\mathrm{H}$ bond activation $\cdot$ Atom economy $\bullet$ Green solvents

[1] A. Ohta, Y. Akita, T. Ohkuwa, M. Chiba, R. Fukunaga, A. Miyafuji, T Nakata, N. Tani, Y. Aoyagi, Heterocycles 1990, 31, 1951-1958.

[2] a) B.-J. Li, S.-D. Yang, Z.-J. Shi, Synlett 2008, 949-957; b) F. Bellina, R. Rossi, Tetrahedron 2009, 65, 10269-10310; c) L. Ackermann, R. Vincente, A. R. Kapdi, Angew. Chem. Int. Ed. 2009, 48, 9792-9826; d) L. Ackermann, Chem. Rev. 2011, 111, 1315-1345; e) N. Kuhl, M. N. Hopkinson, J. Wencel-Delord, F. Glorius, Angew. Chem. Int. Ed. 2012 51, 10236-10254; f) T. Gensch, M. J. James, T. Dalton, F. Glorius, Angew. Chem. Int. Ed. 2018, 57, 2296-2306.

[3] a) R. Rossi, F. Bellina, M. Lessi, C. Manzini, Adv. Synth. Catal. 2014 356, 17-117; b) C. B. Bheeter, L. Chen, J.-F. Soulé, H. Doucet, Cat. Sci. Technol. 2016, 6, 2005-2049.

[4] L. Ackermann, Modern arylation methods, Eds.: Wiley Online Library, 2009.

[5] For selected examples of direct arylations of (benzo)thiophenes: a) J Roger, F. Požgan, H. Doucet, Green Chem. 2009, 11, 425-432; b) B. Liégault, I. Petrov, S. I. Gorlesky, K. Fagnou, J. Org. Chem. 2010, 75, 1047-1060.

[6] For selected examples of direct arylations of (benzo)furans: a) A. Battace, M. Lemhadri, T. Zair, H. Doucet, M. Santelli, Organometallics 2007, 26, 472-474; b) B. Liégaut, D. Lapointe, L. Caron, A. Vlassova, K. Fagnou, J. Org. Chem. 2009, 74, 1826-1834; c) J. J. Dong, J. Roger, F. Požgan, H. Doucet, Green Chem. 2009, 11, 1832-1846; d) Roger, J.; Doucet, H. Eur. J. Org. Chem. 2010, 4412-442; e) D. Roy, S. Mom, S. Royer, D. Lucas, J.-C. Hierso, H. Doucet, ACS Catal. 2012, 2, 10331041 
[7] For selected examples of direct arylations of pyrroles or indoles: a) F. Bellina, S. Cauteruccio, R. Rossi, Eur. J. Org. Chem. 2006, 1379-1382; b) N. Lebrasseur, I. Larrosa, J. Am. Chem. Soc. 2008, 130, 2926-2927; c) R. Jin, K. Yuan, E. Chatelain, J.-F. Soule, H. Doucet, Adv. Synth Catal. 2014, 356, 3831-3841.

[8] For selected examples of direct 2- or 5-arylations of thiazoles: a) A. L. Gottumukkala, H. Doucet, Eur. J. Inorg. Chem. 2007, 3629-3632; b) L.C. Campeau, M. Bertrand-Laperle, J.-P. Leclerc, E. Villemure, S Gorelsky, K. Fagnou, J. Am. Chem. Soc. 2008, 130, 3276; c) D. Lapointe, T. Markiewicz, C. J. Whipp, A. Toderian, K. Fagnou, J. Org Chem. 2011, 76, 749-759; d) S. Bensaid, H. Doucet, ChemSusChem 2012, 5, 1559-1567.

[9] For selected examples of direct arylations of imidazoles and imidazopyridines: a) F. Bellina, S. Cauteruccio, L. Mannina, R. Rossi, S Viel, Eur. J. Org. Chem. 2006, 693-703; b) I. Cerna, R. Pohl, B. Klepetarova, M. Hocek, Org. Lett. 2006, 8, 5389-5392; c) F. Bellina, C Calandri, S. Cauteruccio, R. Rossi, Tetrahedron 2007, 63, 1970-1980; d) F. Bellina, S. Cauteruccio, A. Di Flore, C. Marchietti, R. Rossi, Tetrahedron 2008, 64, 6060-6072; e) J. Roger, H. Doucet, Tetrahedron 2009, 65, 9772-9781; f) H. Y. Fu, L. Chen, H. Doucet, J. Org. Chem. 2012, 77, 4473-4478.

[10] For selected examples of direct arylations of isoxazoles: Y. Fall, C. Reynaud, H. Doucet, M. Santelli, Eur. J. Org. Chem. 2009, 4041-4050.

[11] a) P. T. Anastas, J. C. Warner, Green Chemistry: Theory, Practice, Oxford University Press, New York 1998, p 30; b) T. Welton, Chem. Rev. 1999, 99, 2071-2084; c) P. T. Anastas, M. M. Kirchhoff, Acc Chem. Res. 2002, 35, 686-694

[12] C. Fischmeister, H. Doucet, Green Chem. 2011, 13, 741-753.

[13] For reactions "on water": a) G. L. Turner, J. A. Morris, M. F. Greaney, Angew. Chem. Int. Ed. 2007, 46, 7996-8000; b) S. A. Ohnmacht, P. Mamone, A. J. Culshaw, M. F. Greaney, Chem. Commun. 2008, 1241 1243; c) E. Ferrer Flegeau, M. E. Popkin, M. F. Greaney, Org. Lett. 2008, 10, 2717-2720; d) S. A. Ohnmacht, A. J. Culshaw, M. F. Greaney, Org. Lett. 2010, 12, 224-226; e) L. Joucla, N. Batail, L. Djakovitch, Adv. Synth. Catal. 2010, 352, 2929-2936; f) S. Kalari, D. A. Babar, U. B. Karale, V. B. Makane, H. B. Rode, Tetrahedron Lett. 2017, 58, 28182821; g) For a review: V. Cadierno, J. García-Álvarez, S. E. GarcíaGarrido, Metal-Catalyzed Reactions in Water, P. H. Dixneuf, V. Cadierno Eds.; Wiley-VCH, Weinheim: Germany; 2013, 243-289.

[14] For reaction in polyethylene glycols: L. Ackermann, R. Vicente, Org. Lett. 2009, 11, 4922-4625.

[15] For reaction in carbonates: a) J. Roger, C. Verrier, R. Le Goff, C. Hoarau, H. Doucet, ChemSusChem 2009, 2, 951-956; b) C. Sabah, S. Djebbar, J. F. Soulé, H. Doucet, Chem. Asian J. 2016, 11, 2443-2452.

[16] For reaction in alcohols: S. Bensaid, N. Laidaoui, D. El Abed, S. Kacimi, H. Doucet, Tetrahedron Lett. 2011, 52, 1383-1387.
[17] For reaction in cyclopentyl methyl ether: K. Beydoun, H. Doucet, ChemSusChem 2011, 4, 526-534.

[18] For direct arylation of 1,2,3-triazoles in $\gamma$-Valerolactone: X. Tian, F. Yang, D. Rasina, M. Bauer, S. Warratz, F. Ferlin, L. Vaccaro, L. Ackermann, Chem. Commun. 2016, 52, 9777-9780.

[19] F.-X. Felpin, T. Ayad, S. Mitra, Eur. J. Org. Chem. 2006, 2679-2690.

[20] For direct arylation of isoxazoles with aryl iodides using $\mathrm{Pd} / \mathrm{C}$ as a catalyst: N. Nakamura, Y. Tajima, K. Sakai, Heterocycles 1982, 17, 235-245.

[21] For direct C3-arylation of benzothiophene with aryl chlorides using $\mathrm{Pd} / \mathrm{C}$ associated to $\mathrm{CuCl}$ as a catalyst: D.-T. D. Tang, K. D. Collins, F. Glorius, J. Am. Chem. Soc. 2013, 135, 7450-7453.

[22] For direct C4-arylation of thiophenes with aryliodonium salts as aryl source using Pd/C as a catalyst: D.-T. D. Tang, K. D. Collins, J. B. Ernst, F. Glorius, Angew. Chem. Int. Ed. 2014, 53, 1809-1813.

[23] For direct arylations of heteroaromatics using $\mathrm{Pd}(\mathrm{OH})_{2} / \mathrm{C}$ as catalyst a) M. Parisien, D. Valette, K. Fagnou, J. Org. Chem. 2005, 70, 7578-7584; b) S. Sahnoun, S. Messaoudi, J.-D. Brion, M. Alami, Org. Biomol. Chem. 2009, 7, 4271-4278; c) F. Jafarpour, S. Rahiminejadan, H. Hazrati, J. Org. Chem. 2010, 75, 3109-3112.

[24] For direct arylations of indoles using zeolites, MOFs or nanoparticles: a) G. Cusati, L. Djakovitch, Tetrahedron Lett. 2008, 49, 2499-2502; b) Y. Huang, Z. Lin, R. Cao, Chem. Eur. J. 2011, 17, 12706-12712; c) L. Wang, W.-b. Yi, C. Cai, Chem. Commun. 2011, 47, 806-808; d) P Bizouard, C. Testa, V. A. Zinovyeva, J. Roger, J.-C. Hierso, Synlett 2016, 27, 1227-1231; e) V. A. Zinovyeva, M. A. Vorotyntsev, I. Bezverkhyy, D. Chaumont, J.-C. Hierso, Adv. Funct. Mater. 2011, 21, 1064-1075.

[25] B. Schäffner, F. Schäffner, S. P. Verevkin, A. Börner, Chem. Rev. 2010, 110, 4554-4581.

[26] K. Watanabe, N. Yamagiwa, Y. Torisawa, Org. Process Res. Dev. 2007, 11, 251-258.

[27] For mechanistic studies on Pd/C catalyst for couplings: a) D. A. Conlon, B. Pipik, S. Ferdinand, C. R. LeBlond, J. R. Sawo Jr, B. Izzo, P. Collins, G.-J. Ho, J. M. Williams, Y.-J. Shi, Y. Sun, Adv. Synth. Catal. 2003, 345, 931-935; b) K. D. Collins, R. Honeker, S. Vasquez-Céspedes, D.-T. D. Tang, F. Glorius, Chem. Sci. 2015, 6, 1816-1824.

[28] M. Hird, K. J. Toyne, J. W. Goodby, G. W. Gray, V. Minter, R. P. Tuffin, D. G. McDonnell, J. Mater. Chem. 2004, 14, 1731-1743.

[29] J. Tang, X. Zhao, RSC Adv. 2012, 2, 5488-5490.

[30] A. Gavryushin, C. Kofink, G. Manolikakes, P. Knochel, Tetrahedron 2006, 62, 7521-7533.

[31] B. A. Haag, C. Saemann, A. Jana, P. Knochel, Angew. Chem. Int. Ed. 2011, 50, 7290-7294. 


\section{Entry for the Table of Contents}

\section{FULL PAPER}

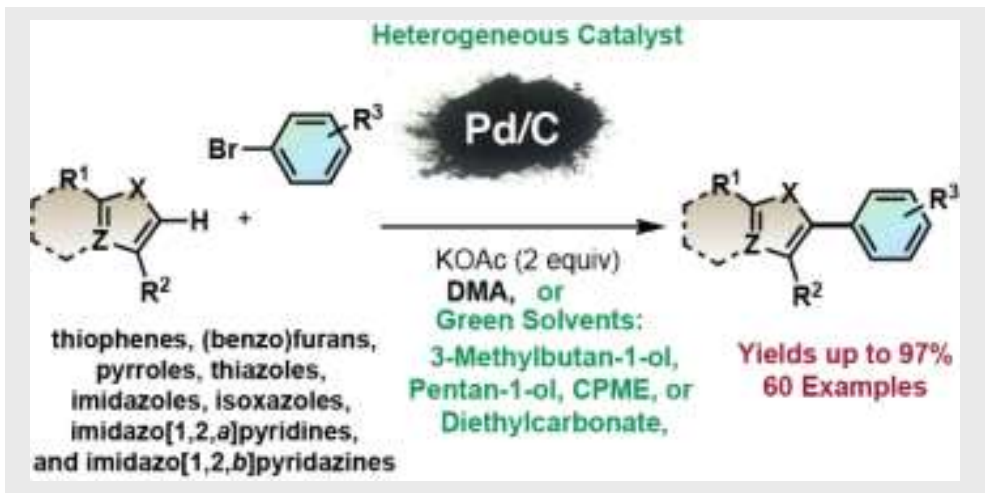

Shuxin Mao, Xinzhe Shi, Jean-François Soulé, * Henri Doucet*

$\mathrm{Pd} / \mathrm{C}$ as Heterogeneous Catalyst for Direct Arylation of Heteroaromatics with Aryl Bromides 